\title{
Review Article \\ Recent Progress in Stability and Stabilization of Systems with Time-Delays
}

\author{
Magdi S. Mahmoud \\ Systems Engineering Department, KFUPM, P.O. Box 5067, Dhahran 31261, Saudi Arabia \\ Correspondence should be addressed to Magdi S. Mahmoud; msmahmoud@kfupm.edu.sa
}

Received 8 November 2016; Accepted 7 March 2017; Published 13 June 2017

Academic Editor: Sabri Arik

Copyright (C) 2017 Magdi S. Mahmoud. This is an open access article distributed under the Creative Commons Attribution License, which permits unrestricted use, distribution, and reproduction in any medium, provided the original work is properly cited.

\begin{abstract}
This paper overviews the research investigations pertaining to stability and stabilization of control systems with time-delays. The prime focus is the fundamental results and recent progress in theory and applications. The overview sheds light on the contemporary development on the linear matrix inequality (LMI) techniques in deriving both delay-independent and delay-dependent stability results for time-delay systems. Particular emphases will be placed on issues concerned with the conservatism and the computational complexity of the results. Key technical bounding lemmas and slack variable introduction approaches will be presented. The results will be compared and connections of certain delay-dependent stability results are also discussed.
\end{abstract}

\section{Introduction}

The occurrence of time-delay phenomenon appears to present many real-world systems and engineering applications. This takes place in either the state, the control input side, or the measurements side. It turns out that delays are strongly involved in challenging areas of communication and information technologies including stabilization of networked controlled systems and high-speed communication networks. In many cases, time-delay is a source of instability. However, for some systems, the presence of delay can have a stabilizing effect. The stability analysis and robust control of time-delay systems (TDS) are, therefore, of theoretical and practical importance.

On the other hand, time-delay systems (TDS) are also termed systems with aftereffect or dead-time, hereditary systems, equations with deviating argument, or differentialdifference equations [1]. As opposed to ordinary differential equations (ODE), TDS belong to the class of functional differential equations (FDE) which are infinite dimension $[2,3]$. A wide variety of dynamical systems can be modeled as time-delay systems [4]. Loosely speaking, time-delay is usually a source of poor performance and instability of a control system. Alternatively, in some few cases, the presence of time-delay is helpful for the stabilization of some systems.
Therefore, stability analysis of time-delay systems is of both practical and theoretical importance [5-9].

A great deal of the basic results is reported in [10-16]. Broadly speaking, stability conditions for time-delay systems can be broadly classified into two categories. One is delayindependent stability conditions and the other is delaydependent stability conditions. Much attention was paid to the study of delay-dependent stability conditions as they yield less conservative results. Recently much work was presented in [17-30] covering alternative issues pertaining to stability and stabilization of dynamical systems with time-delays.

The primary objective of this paper is to

(i) familiarize wider readers with TDS,

(ii) provide a systematic treatment of modern ideas and techniques for researchers.

The paper bridges the huge gap from some basic classical results to recent developments on Lyapunov-based analysis and design with applications to the attractive topics of network-based control and interconnected time-delay control systems. Essentially, it provides an overview on the progress of stability and stabilization of time-delay systems (TDS). Particular emphases will be placed on issues concerned with the conservatism and the computational 
complexity of the results. For simplicity in exposition, the discussions are limited to linear or linearizable systems. Some methods and techniques used to derive stability conditions for time-delay systems are reviewed. Several future research directions on this topic are also discussed.

Notations. Let $\mathbb{R}^{n}$ denote the $n$-dimensional Euclidean space equipped with the norm $\|\cdot\|$. We use $W^{t}, W^{-1}, \lambda_{m}(W)$, and $\lambda_{M}(W)$ to denote, respectively, the transpose, the inverse, the minimum eigenvalue, and the maximum eigenvalue of any square matrix $W$ and $W>0(W<0)$ stands for a symmetrical and positive- (negative-) definite matrix $W$. I stands for unit matrix with appropriate dimension. $\|\alpha\|_{2}^{2}=\sum_{k=0}^{\infty} \alpha^{t}(k) \alpha(k) . \delta H$ denotes the first difference of $H$. We let $\mathbb{R}^{+}$denote the set of nonnegative real numbers; $\mathbb{C}_{n}=\mathbb{C}\left([-h, 0], \mathbb{R}^{n}\right)$ denotes the Banach space of continuous functions $\varphi:[-h, 0] \rightarrow \mathbb{R}^{n}$, and for $\varphi \in \mathbb{C}_{n}$, the associated norm is $\|\varphi\|_{c}=\sup _{-h \leq s \leq 0}\|\varphi\|$. We let $\mathrm{N}=\{1, \ldots, N\}$.

Matrices, if their dimensions are not explicitly stated, are assumed to be compatible for algebraic operations. In symmetric block matrices, we use the symbol $\bullet$ to represent a term that is induced by symmetry. Sometimes, the arguments of a function will be omitted when no confusion can arise.

The following facts are provided in [6].

Fact 1. Let $\Sigma_{1}, \Sigma_{2}, \Sigma_{3}$, and $0<R=R^{t}$ be real constant matrices of compatible dimensions and let $H(t)$ be a real matrix function satisfying $H^{t}(t) H(t) \leq I$. Then for any $\rho>0$ satisfying $\rho \Sigma_{2}^{t} \Sigma_{2}<R$, the following matrix inequality holds:

$$
\begin{gathered}
\left(\Sigma_{3}+\Sigma_{1} H(t) \Sigma_{2}\right) R^{-1}\left(\Sigma_{3}^{t}+\Sigma_{2}^{t} H^{t}(t) \Sigma_{1}^{t}\right) \\
\leq \rho^{-1} \Sigma_{1} \Sigma_{1}^{t}+\Sigma_{3}\left(R-\rho \Sigma_{2}^{t} \Sigma_{2}\right)^{-1} \Sigma_{3}^{t} .
\end{gathered}
$$

Fact 2. For any real matrices $\Sigma_{1}, \Sigma_{2}$, and $\Sigma_{3}$ with appropriate dimensions and $\Sigma_{3}^{t} \Sigma_{3} \leq I$, it follows that

$$
\Sigma_{1} \Sigma_{3} \Sigma_{2}+\Sigma_{2}^{t} \Sigma_{3}^{t} \Sigma_{1}^{t} \leq \alpha^{-1} \Sigma_{1} \Sigma_{1}^{t}+\alpha \Sigma_{2}^{t} \Sigma_{2}, \quad \forall \alpha>0 .
$$

Lemma 1 (Finsler's lemma, [31]). Let $X \in \mathbb{R}^{n}, P=P^{t} \in \mathbb{R}^{n \times n}$, and $H \in \mathbb{R}^{m \times n}$ such that $\operatorname{rank}(H)=r<n$. The following statements are equivalent:

(i) $x^{t} P x<0 \forall H x=\mid 0, \quad x \neq 0$.

(ii) $\left(H^{\perp}\right)^{t} P\left(H^{\perp}\right)<0$.

(iii) $\exists N \in \mathbb{R}^{n \times m}: P+N H+H^{t} N^{t}<0$.

(iv) $\exists \lambda \in \mathbb{R}: P-\lambda H^{t} H<0$.

\section{Overview}

There are many applications where time-delay phenomena appear quite naturally. This includes, but not limited to, the following:

(A) Automotive: combustion model (ignition delay); electromechanical brakes (actuator delay).

(B) Heat exchanger: distributed delay due to conduction in a tube.
(C) Hydraulic networks: the transport phenomenon of water which is modeled as a varying time-delay.

(D) Electrical networks.

(E) Intelligent building: time-delay due to wireless transmission of sensor data.

(F) Marine robotics: transport delay due to sonar measurement of depth.

(G) Population dynamics: predator-prey model based on Volterra model with predator $(y)$ and prey $(x)$ populations ( $t$ is the time-life of prey):

$$
\begin{aligned}
& \dot{x}(t)=r x\left(1-\frac{x(t-\tau)}{K}\right)-\alpha x y, \\
& \dot{y}(t)=-c y+\beta x y .
\end{aligned}
$$

(H) Manufacturing process: the metal cutting process on a lathe which can be described as

$m \ddot{y}(t)+c \dot{y}(t)+k y(t)=-F_{t}[f+y(t)-y(t-\tau)]$.

The study of this model is critical in understanding the regenerative chattering phenomenon.

(I) Epidemics: understanding the dynamics of biological processes and epidemics which is a challenge for health workers engaged in managing treatment strategies. The underlying mechanisms can be revealed by considering epidemics and diseases as dynamical processes, for which the hematology dynamics can be modeled by

$$
\dot{y}(t)=-\lambda y(t)+F[y(t-\tau)]
$$

which formulates the circulating cell populations in one compartment, where $y$ represents the circulating cell population, $\lambda$ is the cell-loss rate, and the monotone function $F$ (describing a feedback mechanism) denotes the flux of cells from the previous compartment. The delay $\tau$ represents the average length of time required to go through the compartment.

(J) Glucose-insulin model: letting $G(t)$ and $I(t)$ represent the levels of plasma glycemia and insulinemia; then

$$
\begin{aligned}
& \dot{G}(t)=K_{x g i} G(t) I(t)+\frac{T_{g h}}{V_{G}}, \\
& \dot{I}(t)=-K_{x i} I(t)+\frac{T_{i G_{\max }}}{V_{I}} f\left[G\left(t-\tau_{g}\right)\right],
\end{aligned}
$$

where

(i) $K_{x g i}$ is rate of glucose uptake by tissues (insulindependent) per pM of plasma insulin concentration,

(ii) $T_{g h}$ is net balance between hepatic glucose output and insulin-independent zero-order glucose tissue uptake (mainly by the brain), 
(iii) $V_{G}$ is apparent distribution volume for glucose,

(iv) $K_{x i}$ is apparent first-order disappearance rate constant for insulin,

(v) $T_{i G_{\max }}$ is maximal rate of second-phase insulin release,

(vi) $V_{I}$ is apparent distribution volume for insulin,

(vii) $\tau_{g}$ is apparent delay with which the pancreas varies secondary insulin release in response to varying plasma glucose concentrations,

(viii) $f(\cdot)$ is nonlinear function that models the Insulin Delivery Rate.

(K) Neutral delay systems: arising, for instance, in the analysis of the coupling between transmission lines and population dynamics: evolution of forests. The model is based on a refinement of the delay-free logistic (or Pearl-Verhulst equation) where effects as soil depletion and erosion have been introduced

$$
\dot{x}(t)=r x(t)\left[1-\frac{x(t-\tau)+c \dot{x}(t-\tau)}{K}\right],
$$

where $x$ is the population, $r$ is the intrinsic growth rate, and $K$ is the environmental carrying capacity.

\section{Models and Solutions}

A general model of TDS can be expressed as

$$
\begin{aligned}
& \dot{x}(t)=f\left(x_{t}, t, u_{t}\right), \quad t \geq t_{o} \\
& y(t)=g\left(x_{t} ; t ; u_{t}\right),
\end{aligned}
$$

where

$$
\begin{gathered}
x_{t}(\theta)=x(t+\theta) ; \quad-h \leq \theta \leq 0, \\
u_{t}(\theta)=u(t+\theta) ; \quad-h \leq \theta \leq 0, \\
x(\theta)=\varphi(\theta) ; \quad t_{0}-h \leq \theta \leq t_{0} .
\end{gathered}
$$

3.1. Retarded Systems. It is quite natural to consider, as statespace, the set $\mathbb{S}=\mathbb{S}\left([-h ; 0] ; \mathbb{R}^{n}\right)$ of continuous functions mapping the interval $[-h ; 0] \rightarrow \mathbb{R}^{n}$, with the topology of uniform convergence. The initial condition $\varphi(\theta)$ must be prescribed as $\Phi:[-h ; 0] ; \mathbb{R}^{n}$. Observe that $\Phi \in \mathbb{C}$ or may involve bounded jumps at some discontinuity instants. The nature of the solution (and of its initial value) then distinguishes FDE from ODE.

Definition 2 (see [3]). A function $x$ is said to be a solution on $[\sigma-h ; \sigma+a]$ of the retarded functional differential equation (RDE)

$$
\begin{aligned}
& \dot{x}(t)=f\left(t, x_{t}\right), \\
& f: \Omega \subset \mathbb{R} \times \mathbb{S} \rightarrow \mathbb{R}^{n},
\end{aligned}
$$

if there are $\sigma \in \mathbb{R}$ and $a>0$ such that $x \in \mathbb{S}\left([\sigma-h ; \sigma+h] ; \mathbb{R}^{n}\right)$, $\left(t, x_{t}\right) \in \Omega$, and $x(t)$ satisfies (10) for $t \in[\sigma-h ; \sigma+a]$. For given $\sigma \in \mathbb{R} ; \varphi \in \mathbb{S}$, we say that $x(\sigma ; \varphi ; f)$ is a solution of (10) with initial value $\varphi$ at $\sigma$ or simply a solution through $(\sigma ; \varphi)$ if there is $a>0$ such that $x(\sigma ; \varphi ; f)$ is a solution of $(10)$ on $t \in[\sigma-h ; \sigma+a]$ and $x_{\sigma}(\sigma ; \varphi ; f)=\varphi$.

Supposing that $\Omega$ is open and $f \in \mathbb{S}\left(\Omega, \mathbb{R}^{n}\right)$, then a function $x \in \mathbb{S}\left([\sigma-h-\alpha ; \sigma] ; \mathbb{R}^{n}\right), \alpha>0$, is referred to as a backward continuation of the solution through $(\sigma ; \varphi)$ if $x_{\sigma}=\varphi$ and for any $\sigma_{1} \in[\sigma-\alpha, \sigma] ;\left(\sigma_{1} ; x_{\sigma_{1}}\right) \in \Omega$ and $x$ is a solution of (10) on $\left(\sigma_{1}-h ; \sigma\right)$ through $\left(\sigma_{1} ; x_{\sigma_{1}}\right)$.

The interested reader is referred to [3] for further useful discussions.

3.2. Neutral Systems. Neutral systems also are delay systems but involve the same highest derivation order for some components of $x(t)$ at both time $t$ and past time(s) $t^{o}<t$, which implies an increased mathematical complexity. Neutral systems are represented by

$$
\dot{x}(t)=f\left(x_{t}, t, \dot{x}_{t}, u_{t}\right)
$$

or

$$
\frac{d \mathbf{F} x_{t}}{d t}=f\left(x_{t}, t, u_{t}\right)
$$

where $\mathbf{F}: \mathbb{S} \rightarrow \mathbb{R}^{n}$ is a regular operator with deviating argument in time, as, for instance, with $D$ constant matrix

$$
\mathbf{F} x_{t}=x(t)-D x(t-\omega) \text {. }
$$

It is significant to observe that the solutions of retarded systems have their differentiability degree smoothed with increasing time, but this is no longer true for neutral systems due to the implied difference-equation involving $\dot{x}(t)$; the trajectory may replicate any irregularity of the initial condition $\varphi(t)$, even if $f$ and $F$ satisfy many smoothness properties.

3.3. Models for Linear Time-Invariant Systems. In the linear, time-invariant case (LTI), the corresponding general timedelay model is

$$
\begin{aligned}
\dot{x}(t)= & \sum_{\ell=1}^{p} D_{\ell} \dot{x}\left(t-\omega_{\ell}\right) \\
& +\sum_{j=0}^{q}\left[A_{j} x\left(t-h_{j}\right)+B_{j} u\left(t-h_{j}\right)\right] \\
& +\sum_{m=1}^{r} \int_{t-\tau_{m}}^{t}\left[G_{m}(\theta) x(\theta)+H_{m}(\theta) u(\theta)\right] d \theta, \\
y(t)= & \sum_{j=0}^{q} C_{j} x\left(t-h_{j}\right)+\sum_{m=1}^{r} \int_{t-\tau_{m}}^{t} N_{m}(\theta) x(\theta) d \theta,
\end{aligned}
$$

where

(i) $h_{0}=0$ and $A_{0}$ is constant instantaneous matrix;

(ii) constant matrices $A_{j}$; $j>0$ represent discrete-delay phenomena; 
(iii) the sum of integrals corresponds to distributed delay effects, weighted by $G_{m}$ over the time intervals $[t-$ $\left.\tau_{m} ; t\right]$

(iv) matrices $D_{\ell}$ account for the neutral part;

(v) matrices $B_{j}$ and $H_{m}(s)$ are input matrices;

(vi) in brief, $h=\max _{j, m, \ell}\left\{h_{j} ; \tau_{m} ; \omega_{\ell}\right\}$.

Note that (15), $y(t) \in \mathbb{R}^{p}$, represents the output description, with discrete $C_{j}$ and distributed $N_{m}(\theta)$ delayed parts as well. The special case of (14)-(15)

$$
\begin{aligned}
\dot{x}(t) & =\sum_{j=0}^{q}\left[A_{j} x\left(t-h_{j}\right)+B_{j} u\left(t-h_{j}\right)\right], \\
h_{0} & =0<h_{1}<\cdots, h_{q-1}<h_{q}
\end{aligned}
$$

has been investigated extensively in the literature.

\section{Notion of Stability}

As a starting point, we recall the following stability notion for time-delay system (3).

Definition 3. If, for any $t_{0} \in \mathbb{R}$ and any $\varepsilon>0$, there exists a $\delta=\delta\left(t_{0}, \varepsilon\right)>0$ such that $\left\|x_{t_{0}}\right\|_{c}<\delta$ implies $\|x(t)\|<\varepsilon$ for all $t \geq t_{0}$, then the trivial solution of time-delay system (3) is stable.

The following properties are readily recognized.

(i) If the trivial solution of time-delay system (3) is stable and if $\delta$ can be chosen independently of $t_{0}$, then the trivial solution of time-delay system (3) is uniformly stable.

(ii) If the trivial solution of time-delay system (3) is stable and if, for any $t_{0} \in \mathbb{R}$ and any $\varepsilon>0$, there exists $\delta_{a}=\delta_{a}\left(t_{0}, \varepsilon\right)>0$ such that $\left\|x_{t_{0}}\right\|_{c}<\delta_{a}$ implies $\lim _{t \rightarrow \infty} x(t)=0$, then the trivial solution of timedelay system (3) is asymptotically stable.

(iii) If the trivial solution of time-delay system (3) is uniformly stable and there exists $\delta_{a}>0$, such that $\left\|x_{t_{0}}\right\|_{c}<\delta_{a}$ implies $\|x(t)\|<\eta$ for $t \geq t_{0}+T$ and $t_{0} \in \mathbb{R}$, then the trivial solution of time-delay system (3) is uniformly asymptotically stable.

(iv) If the trivial solution of time-delay system (3) is (uniformly) asymptotically stable and if $\delta_{a}$ can be arbitrarily large but finite number, then the trivial solution of time-delay system (3) is globally (uniformly) asymptotically stable.

\section{Fundamental Stability Theorems}

In the study of stability analysis of time-delay systems, the methods of Lyapunov functions and Lyapunov-Krasovskii functionals play important roles. There are two Lyapunov methods are often used:

(A) Lyapunov-Krasovskii functional (LKF) method,

(B) Lyapunov-Razumikhin function (LRF) method.
It is significant to observe that LKF method deals with functionals which essentially have scalar values whereas Lyapunov-Razumikhin function (LRF) method involves only functions rather than functionals.

In this section, these two methods are reviewed; see [6] for details.

Consider the following time-delay system described by

$$
\dot{x}(t)=f\left(t, x_{t}\right), \quad t \geq t_{o},
$$

where

(i) $x_{t}=x(t+\theta) ;-\theta_{m} \leq \theta \leq 0$,

(ii) $f ; \mathbb{R} \times \mathbb{C}_{n} \rightarrow \mathbb{R}^{n}$ is continuous and is Lipschitz in $x_{t}$,

(iii) $f(t, 0)=0$.

In the sequel, we let $x_{t}(s, \varphi)$ be the solution of (17) at time $t$ with initial condition $x_{s}=\varphi$. Let $\mathbb{G}$ be a bounded subset of $\mathbb{C}_{n}$ and let $\mathbb{H}$ be a bounded subset of $\mathbb{R}^{n}$.

A statement of Lyapunov-Krasovskii stability method is provided by the following theorem.

Theorem 4. Suppose that $f$ maps $\mathbb{R} \times \mathbb{G}$ into $\mathbb{H}$ and $u, v, w$ : $\mathbb{R}^{+} \rightarrow \mathbb{R}^{+}$are continuous, nondecreasing functions with $u(0)=$ $v(0)=0$ and $u(\beta)>0$ and $v(\beta)>0$, for $\beta>0$. If there exists $a$ continuous functional $\mathbb{V}: \mathbb{R} \times \mathbb{C}_{n} \rightarrow \mathbb{R}$ such that

(1) $u(\|\varphi(0)\|) \leq \mathbb{V}(t, \varphi) \leq v(\|\varphi(0)\|)$,

(2) $\dot{\mathbb{V}}(t, \varphi) \leq-w(\|\varphi(0)\|)$,

where

$$
\begin{aligned}
\dot{\mathbb{V}} & (t, \varphi) \\
& =\lim _{\Delta t \rightarrow 0^{+}} \frac{1}{\Delta t}\left(\mathbb{V}\left(t+\Delta t, x_{t+\Delta t}(t, \varphi)\right)-\mathbb{V}(t, \varphi)\right),
\end{aligned}
$$

then the trivial solution of time-delay system (3) is uniformly stable. If $w(\beta)>0$, for $\beta>0$, then the trivial solution of time-delay system (3) is uniformly asymptotically stable. Additionally, if $\lim _{\beta \rightarrow \infty} u(\beta) \rightarrow \infty$, then the trivial solution of time-delay system (3) is globally uniformly asymptotically stable.

In some cases, the LKF involving terms depending on the state derivatives $\dot{x}_{t}$ are quite effective in the derivation of the stability conditions. This will in turn requires the modification of the conditions in Theorem 4. See [8] for details.

A statement of Lyapunov-Razumikhin stability method is provided by the following theorem.

Theorem 5. Suppose that $f$ maps $\mathbb{R} \times \mathbb{G}$ into $\mathbb{H}$ and $u, v, w$ : $\mathbb{R}^{+} \rightarrow \mathbb{R}^{+}$are continuous, nondecreasing functions with $u(0)=$ $v(0)=0$ and $u(\beta)>0$ and $v(\beta)>0$, for $\beta>0$, and $v$ is strictly increasing. If there exists a continuous functional $\mathbb{V}: \mathbb{R} \times \mathbb{R}^{n} \rightarrow$ $\mathbb{R}$ such that

$$
\begin{aligned}
& \text { (1) } u(\|x\|) \leq \mathbb{V}(t, x) \leq v(\|x\|), \\
& \text { (2) } \dot{\mathbb{V}}(t, \varphi) \leq-w(\|\varphi(0)\|), \text { if } \\
& {[\mathbb{V}(t+\theta, x(t+\theta)) \leq \mathbb{V}(t, x(t))], \text { for } \theta \in[-h, 0],}
\end{aligned}
$$


where

$$
\begin{aligned}
\dot{\mathbb{V}}(t, x(t)) & =\frac{d}{d t} \mathbb{V}(t, x(t)) \\
& =\frac{\partial \mathbb{V}(t, x(t))}{\partial t}+\frac{\partial \mathbb{V}(t, x(t))}{\partial x} f\left(t, x_{t}\right),
\end{aligned}
$$

then the trivial solution of time-delay system (3) is uniformly stable. If $w(\beta)>0$, for $\beta>0$, there exists a continuous nondecreasing function $q(\beta)>0$, for $\beta>0$, and the foregoing condition (2) is strengthened to $\dot{\mathbb{V}}(t, x(t)) \leq-w(\|x(t)\|)$, if

$$
(\mathbb{V}(t+\theta, x(t+\theta)) \leq q(\mathbb{V}(t, x(t)))),
$$

$$
\text { for } \theta \in[-h, 0] \text {, }
$$

then the trivial solution of time-delay system (3) is uniformly asymptotically stable. Additionally, if $\lim _{\beta \rightarrow \infty} u(\beta) \rightarrow \infty$, then the trivial solution of time-delay system (3) is globally uniformly asymptotically stable.

The following Halanay result [7] also plays an important role in the stability analysis of time-delay systems.

Theorem 6. Suppose that constant scalars $k_{1}$ and $k_{2}$ satisfy $k_{1}>0, k_{2}>0$, and $y(t)$ is a nonnegative continuous function on $\left[t_{0}-\tau, t_{0}\right]$ satisfying

$$
\begin{aligned}
& \dot{y}(t) \leq-k_{1} y(t)+k_{2} \bar{y}(t), \quad t \geq t_{0}, \\
& \bar{y}(t)=\sup _{t-\tau \leq s \leq t}\{y(s)\}, \quad \tau \geq 0 .
\end{aligned}
$$

Then, for $t \geq t_{0}$, one has

$$
y(t) \leq \bar{y}\left(t_{0}\right) \exp \left(-\kappa\left(t-t_{0}\right)\right),
$$

where $\kappa>0$ is the unique solution to the following equation:

$$
\kappa=k_{1}-k_{2} \exp (-\kappa \tau) \text {. }
$$

Remark 7. Theorems 4 through 6 can be used to derive stability conditions for the case when the delay is time-varying, which is continuous but not necessarily differentiable.

Remark 8 . In the sequel, stability conditions for time-delay systems can be broadly classified into two types:

(1) Delay-independent stability (DIS) conditions which do not include information about the delay. Generally speaking, DIS conditions are simpler to apply.

(2) Delay-dependent stability (DDS) conditions which involve information on the size and pattern of the delay. DDS conditions are less conservative especially in the case when the time-delay is small.

In the sequel, this paper focuses on the delay-dependent stability problem and the objective is twofold:

(A) to develop delay-dependent conditions to provide a maximal allowable delay as large as possible,
(B) to develop delay-dependent conditions by using as few as possible decision variables while keeping the same maximal allowable delay.

Alternatives approaches were proposed in the literature to obtain DDS conditions, among which the linear matrix inequality (LMI) approach is the most popular. The LMI approach has played a significant role due to the fact that family linear matrix inequalities can be readily converted into a convex optimization problem. The latter can be handled efficiently by resorting to recently developed numerical algorithms for solving LMIs [31]. Additional reason that makes LMI conditions appealing is their frequent readiness to solve the corresponding synthesis problems once the stability (or other performance) conditions are established, especially when state feedback is employed.

\section{Stability Results for Linear Delay Systems}

For the sake of simplicity, the following linear system with a single discrete delay is considered:

$$
\dot{x}(t)=A x(t)+A_{d}(t-\tau(t)), \quad t \geq t_{0},
$$

where $x(t) \in \mathbb{R}^{n}$ is the state vector, $A$ and $A_{d}$ are system matrices with appropriate dimensions, and $\tau(t)$ is the timedelay factors. There are several classes of time-delay patterns considered in the literature as follows:

Class A: constant delay,

$$
\tau(t)=d, \quad \forall t .
$$

Class B: unknown-but-bounded delay,

$$
0<\tau(t) \leq \tau_{M}, \quad \forall t .
$$

Class C: bounded time-varying delay,

$$
\tau_{m} \leq \tau(t) \leq \tau_{M}, \quad \forall t .
$$

Class D: bounded time-varying delay with bounded derivative,

$$
\begin{array}{ll}
\tau_{m} \leq \tau(t) \leq \tau_{M}, & \forall t \\
d_{m} \leq \dot{\tau}(t) \leq d_{M}, & \forall t .
\end{array}
$$

6.1. Constant Delay. When the time-delay is constant, the system described by (30) can be rewritten as

$$
\dot{x}(t)=A_{0} x(t)+A_{d} x(t-d), \quad t \geq t_{0} .
$$

Natural extensions of the quadratic Lyapunov functions can be particularly used to study in the framework of LTI delay systems (30) and the functional

$$
\mathbb{V}_{0}\left(x_{t}\right)=x^{t}(t) \mathscr{P} x(t)+\int_{-d}^{0} x^{t}(t+\theta) \mathscr{Q} x(t+\theta) d \theta .
$$

One obtains sufficient conditions by the following theorem. 
Theorem 9. The time-delay system (30) is asymptotically stable for any $d \geq 0$ if there exist matrices $\mathscr{P}>0$ and $\mathscr{Q}>0, \mathscr{R}$ verifying

$$
\mathscr{P} A_{0}+A_{0}^{t} \mathscr{P}+\mathscr{P} A_{d} \mathscr{Q}^{-1} A_{d}^{t} \mathscr{P}+\mathscr{Q}+\mathscr{R}=0
$$

or equivalently the LMI

$$
\left[\begin{array}{cc}
\mathscr{P} A_{0}+A_{0}^{t} \mathscr{P}+Q & \mathscr{P} A_{d} \\
\bullet & -Q
\end{array}\right]<0 .
$$

It is significant to observe in the delay-free case, $A_{d}=0$, that (33) provides the link with the Lyapunov equation for ODE. Nevertheless, in the delayed case $A_{d} \neq 0$, this sufficient condition is far from being necessary. From here, many generalizations were proposed, involving different alternative terms:

$$
\begin{aligned}
& \mathbb{V}_{1}\left(x_{t}\right)=x^{t}(t) \mathscr{P} x(t), \\
& \mathbb{V}_{2}\left(x_{t}\right)=x^{t}(t) \int_{-d_{j}}^{0} Q_{j} x(t+\theta) d \theta, \\
& \mathbb{V}_{3}\left(x_{t}\right)=\int_{-d_{j}}^{0} x^{t}(t+\theta) \mathcal{S}_{j} x(t+\theta) d \theta,
\end{aligned}
$$

$$
\begin{aligned}
& \mathbb{V}_{4}\left(x_{t}\right)=\int_{-\tau_{j}}^{0} \int_{t+\theta}^{0} x^{t}(\theta) \mathscr{R}_{j} x(\theta) d \theta d s, \\
& \mathbb{V}_{5}\left(x_{t}\right)=x^{t}(t) \int_{-d_{j}}^{0} \mathscr{P}_{j}(\eta) x(t+\eta) d \eta, \\
& \mathbb{V}_{6}\left(x_{t}\right)=\int_{-d_{j}}^{0} \int_{-d_{j}}^{0} x^{t}(t+\eta) \mathscr{P}(\eta, \theta) x(t+\theta) d \eta d \theta .
\end{aligned}
$$

The following points are noteworthy:

(1) Loosely speaking, the terms $\mathbb{V}_{2} ; \mathbb{V}_{3}$ are used for the delay-independent stability of discrete delays.

(2) The term $V_{4}$ is meant for distributed delays or discrete-delay dependent stability. On considering system (30) along with

$$
\mathbb{V}\left(x_{t}\right)=\mathbb{V}_{1}(x(t))+\mathbb{V}_{4}\left(x_{t}\right)+\mathbb{V}_{4}\left(x_{t-h}\right)
$$

standard manipulation leads, with $R_{1}$ for $V_{4}\left(x_{t}\right) ; R_{2}$ for $V_{4}\left(x_{t}-h\right)$, to the following delay-dependent LMI condition:

$$
\left[\begin{array}{ccc}
\mathscr{P}\left(A_{0}+A_{d}\right)+\left(A_{0}+A_{d}\right)^{t} \mathscr{P}+d \mathscr{R}_{1}+d \mathscr{R}_{2} & d \mathscr{P} A_{d} A_{0} & h \mathscr{P} A_{d}^{2} \\
\bullet & -d \mathscr{R}_{1} & 0 \\
\bullet & \bullet & -d \mathscr{R}_{2}
\end{array}\right]<0 .
$$

(3) Although the terms $V_{5}$ and $V_{6}$ appear, in a general form, in necessary and sufficient schemes (see [1012]), the general computation of the time-varying matrices is excessively burden. To avoid such computational limitations, a discretization scheme incorporating piecewise-constant functions $P_{j}(:)$ was introduced in $[15,16]$.

6.2. Time-Varying Delay. In what follows, we will review the LMI techniques in deriving DDS results for the single-delay case. Extension to the multiple-delay case is a straightforward task. We consider the class of time-delay systems (class B) in which the delay factor is continuous but bounded.

$$
\begin{aligned}
\dot{x}(t)=A_{0} x(t)+A_{d} x(t-\tau(t)) & \\
x(t) & =\varphi(t), t \in\left[-\tau_{M}, 0\right] .
\end{aligned}
$$

Similar to (31), we consider the LKF of the form

$$
\begin{aligned}
\widehat{V}_{0}\left(x_{t}\right)= & x^{t}(t) \mathscr{P} x(t) \\
& +\int_{t-\tau(t)}^{t} x^{t}(t+\theta) \mathscr{Q} x(t+\theta) d \theta .
\end{aligned}
$$

Since the time-varying delay $\tau(t)(t)$ may not be differentiable, we introduce the following equalities for any matrices $\mathscr{Y}, \mathscr{W}$, and $\mathcal{S}$ with appropriate dimensions:

$$
\begin{array}{r}
\dot{x}^{t}(t) \mathcal{Y}\left[A_{0} x(t)+A_{d} x(t-\tau(t))-\dot{x}(t)\right]=0, \\
x^{t}(t) \mathscr{W}\left[A_{0} x(t)+A_{d} x(t-\tau(t))-\dot{x}(t)\right]=0, \\
\dot{x}^{t}(t-\tau(t)) \mathcal{S}\left[A_{0} x(t)+A_{d} x(t-\tau(t))-\dot{x}(t)\right]=0 .
\end{array}
$$

The following theorem summarized the main result.

Theorem 10. The time-delay system (37) is asymptotically stable if there exist matrices $\mathscr{P}>0, \mathscr{Y}, \mathscr{W}$, and $\mathcal{S}$ such that

$$
\left[\begin{array}{ccc}
\mathscr{W} A_{0}+A_{0}^{t} \mathscr{W}^{t} & \mathscr{W} A_{d}+A_{0}^{t} \mathcal{S}^{t} & A_{0}^{t} \mathscr{Y}^{t}+\mathscr{P}-\mathscr{W} \\
\bullet & \mathcal{S} A_{d}+A_{d}^{t} \delta^{t} & A_{d}^{t} \mathscr{Y}^{t}-\mathcal{S} \\
\bullet & \bullet & -\mathcal{Y}-\mathscr{Y}^{t}
\end{array}\right]
$$


Consider the time-delay system

$$
\begin{aligned}
& \dot{x}(t)=A_{0} x(t)+A_{d} x(t-\tau(t)), \\
& \qquad x(t)=\varphi(t), t \in\left[-\tau_{M}, 0\right], \\
& 0 \leq \tau_{m} \leq \tau(t) \leq \tau_{M}, \\
& \sigma \leq \dot{\tau}(t) \leq \mu .
\end{aligned}
$$

According to the Lyapunov-Razumikhin stability method Theorem 5, the following stability condition can be obtained.

Theorem 11. The time-delay system (41) is asymptotically stable if there exist matrix $\mathscr{P}>0$ and a scalar $\sigma>0$ such that

$$
\left[\begin{array}{cc}
\mathscr{P} A_{0}+A_{0}^{t} \mathscr{P}+\sigma \mathscr{P} & \mathscr{P} A_{d} \\
\bullet & -\sigma \mathscr{P}
\end{array}\right]<0 .
$$

On choosing the LKF (31), a delay-independent stability condition can be derived in the following form.

Theorem 12. The time-delay system (41) is asymptotically stable if there exist matrices $\mathscr{P}>0$ and $\mathbb{Q}>0$ such that

$$
\left[\begin{array}{cc}
\mathscr{P} A_{0}+A_{0}^{t} \mathscr{P}+\mathscr{Q} & \mathscr{P} A_{d} \\
\bullet & -(1-\mu) \mathscr{Q}
\end{array}\right]<0 .
$$

Remark 13. It should be noted that Theorem 12 is independent of the time-delay and therefore is very conservative especially when the time-delay is small. When the delay is constant, $\tau(t) \equiv d$, it follows from the Schur complements that $(40)$ is equivalent to

$$
\mathscr{P} A_{0}+A_{0}^{t} \mathscr{P}+\mathscr{Q}+\mathscr{P} A_{d} Q^{-1} A_{d} \mathscr{P}<0 .
$$

In turn this implies that

$$
\mathscr{P}\left(A_{0}+A_{d}\right)+\left(A_{0}+A_{d}\right)^{t} \mathscr{P}<0
$$

which is a necessary and sufficient condition for the stability of system (41) with zero delay.

In the literature, the following Lyapunov functional is often used to derive delay-dependent results.

$$
\begin{aligned}
\mathbb{V}\left(t, x_{t}\right)= & x^{t}(t) \mathscr{P} x(t)+\int_{t-\tau(t)}^{t} x^{t}(s) \mathcal{Q} x(s) d s \\
& +\int_{-\tau_{M}}^{0} \int_{t+\theta}^{t} \dot{x}^{t}(s) \mathscr{X} \dot{x}(s) d s d \theta .
\end{aligned}
$$

It was first introduced in $[32,33]$. Using the free-weighting [34], the following DDS condition can be derived based on the LKF (47).

Theorem 14. The time-delay system (41) is asymptotically stable if there exist matrices $\mathscr{P}>0, \mathbb{Q}>0, \mathscr{Z}>0$, and
$\left[\begin{array}{cc}X_{11} & X_{12} \\ \bullet & X_{22}\end{array}\right] \geq 0$, and any matrices $M$ and $N$ of appropriate dimensions such that

$$
\begin{gathered}
{\left[\begin{array}{ccc}
\mathbb{L}_{11} & \mathbb{L}_{12} & \tau_{M} A_{0}^{t} \mathscr{Z} \\
\bullet & \mathbb{L}_{22} & \tau_{M} A_{d}^{t} \mathscr{Z} \\
\bullet & \bullet & -\tau_{M} \mathscr{Z}
\end{array}\right]<0,} \\
{\left[\begin{array}{ccc}
X_{11} & X_{12} & M \\
\bullet & X_{22} & N \\
\bullet & \bullet & \mathscr{Z}
\end{array}\right] \geq 0,}
\end{gathered}
$$

where

$$
\begin{aligned}
& \mathbb{L}_{11}=\mathscr{P} A_{0}+A_{0}^{t} \mathscr{P}+M+M^{t}+\mathscr{Q}+\tau_{M} X_{11}, \\
& \mathbb{L}_{12}=\mathscr{P} A_{d}+M+N^{t}+\tau_{M} X_{12}, \\
& \mathbb{L}_{22}=-N-N^{t}-(1-\mu) \mathcal{Q}+\tau_{M} X_{22} .
\end{aligned}
$$

6.3. Augmented Lyapunov Functional. Recalling that the first term in most LKFs is $x^{t}(t) P x(t)$ which involves the current state $x(t)$ only and does not reflect the delayed state. Hence, an augmented Lyapunov functional was proposed in [35] for system described by (30).

$$
\begin{aligned}
\mathbb{V}\left(t, x_{t}\right)= & \xi^{t}(t) \mathscr{P} \xi(t)+\int_{t-d}^{t} \varrho^{t}(s) \mathscr{Q} \varrho(s) d s \\
& +\int_{-d}^{0} \int_{t+\theta}^{t} \varrho^{t}(s) \mathscr{Z} \varrho(s) d s d \theta, \\
\xi^{t}(t)= & {\left[\begin{array}{ll}
x^{t}(t) & x^{t}(t-d) \int_{t-d}^{t} x^{t}(s) d s
\end{array}\right], } \\
\varrho^{t}(s)= & {\left[\begin{array}{ll}
x^{t}(s) & \left.\dot{x}^{t}(s)\right] .
\end{array}\right.}
\end{aligned}
$$

Remark 15. Compared with the Lyapunov functional (47), the augmented Lyapunov functional can lead to less conservative results. Additionally, it is also applicable for systems with time-varying delay, which can be seen in [36] and references therein.

6.4. Triple Integral Lyapunov Functional. On examining the LKFs (31) and (50), it can be seen that the Lyapunov functional often contains integral terms: single $\int_{t-\tau(t)}^{t} x^{t}(t+$ $\theta) Q x(t+\theta) d \theta$ and double $\int_{-d}^{0} \int_{t+\theta}^{t} \varrho^{t}(s) \mathscr{Z} \varrho(s) d s d \theta$ in order to bring the effect of time-delays.

A natural question which arose is whether introducing triple integral terms in the Lyapunov functional would yield improvement in the stability behavior. This question 
is addressed $[37,38]$ by extending the LKFs (50)-(51) and incorporating a triple integral term to yield the form

$$
\begin{aligned}
\mathbb{V}\left(t, x_{t}\right)= & \xi^{t}(t) \mathscr{P} \xi(t)+\int_{t-d}^{t} \varrho^{t}(s) \mathscr{Q} \varrho(s) d s \\
& +\int_{-d}^{0} \int_{t+\theta}^{t} \varrho^{t}(s) \mathscr{X} \varrho(s) d s d \theta \\
& +\int_{-d}^{0} \int_{\theta}^{0} \int_{t+\beta}^{t} \dot{x}^{t}(s) \mathscr{R} \dot{x}(s) d s d \beta d \theta .
\end{aligned}
$$

Remark 16. It is reported in $[37,38]$ by simulation results that the Lyapunov functional containing triple integral terms is quite effective in reduction of the conservatism of the stability conditions.

6.5. Newton-Leibniz Formula. An alternative route can be pursued by using the Newton-Leibniz formula

$$
\begin{aligned}
x(t-d) & =x(t)-\int_{t-d}^{t} \dot{x}(\alpha) d \alpha \\
& =x(t)-\int_{t-d}^{t}\left[A_{0} x(\alpha)+A_{d} x(\alpha-d)\right] d \alpha
\end{aligned}
$$

and recalling (30) to yield

$$
\begin{aligned}
\dot{x}(t)= & {\left[A_{0}+A_{d}\right] x(t) } \\
& -A_{d} \int_{t-d}^{t}\left[A_{0} x(\alpha)+A_{d} x(\alpha-d)\right] d \alpha .
\end{aligned}
$$

Remark 17. It should be clear that the asymptotic stability of the time-delay system in (54) implies that of system (30).

Following [6], we proceed to study the DDS of system (54) using the following LKF candidate:

$$
\begin{aligned}
& \mathbb{V}\left(t, x_{t}\right)= x^{t}(t) \mathscr{P}^{-1} x(t) \\
&+\int_{-h}^{0} \int_{t+\alpha}^{t} x^{t}(\theta) A_{d}^{t} Q_{1}^{-1} A_{d} x(\theta) d \theta d \alpha \\
&+\int_{-h}^{0} \int_{t-h+\alpha}^{t} x^{t}(\theta) A_{d}^{t} Q_{2}^{-1} A_{d} x(\theta) d \theta d \alpha, \\
& \mathscr{P}>0, \mathbb{Q}_{1}>0, \mathbb{Q}_{2}>0 .
\end{aligned}
$$

Define

$$
\begin{aligned}
\Gamma= & \mathscr{P}\left(A_{0}+A_{d}\right)+\left(A_{0}+A_{d}\right)^{t} \mathscr{P} \\
& +A_{d}\left(Q_{1}+Q_{2}\right) A_{d}^{t} .
\end{aligned}
$$

The main stability result is established by the following theorem.
Theorem 18. The time-delay system (54) is asymptotically stable for any delay satisfying $0<d \leq d_{M}$ if there exist matrices $\mathscr{P}>0, Q_{1}$, and $\mathbb{Q}_{2}$ such that

$$
\left[\begin{array}{ccc}
\Gamma & d_{M} \mathscr{P} A_{0}^{t} & d_{M} \mathscr{P} A_{d}^{t} \\
\bullet & -Q_{1} & 0 \\
\bullet & \bullet & -Q_{2}
\end{array}\right]<0 .
$$

Remark 19. The technique by using the Newton-Leibniz formula to transform the time-delay system to appropriate for DDS analysis is quite useful. However, still a different route of writing (54) would be

$$
\begin{aligned}
& \dot{x}(t)=\left[A_{0}+A_{d}\right] x(t)-A_{d} \int_{t-d}^{t} \dot{x}(d \alpha) d \alpha, \\
& \frac{d}{d t}\left[x(t)+A_{d} \int_{t-d}^{t} x(\alpha) d \alpha\right]=\left(A_{0}+A_{d}\right) x(t) .
\end{aligned}
$$

However, all the transformed time-delay systems by using the Newton-Leibniz formula introduce additional dynamics which may cause conservatism as the delay-dependent conditions derived based on the transformed systems.

6.6. Bounding Techniques. In studying delay-dependent stability for time-delay systems, it is desirable to find methods that yield stability conditions with reduced conservatism. A wide class of early methods rely on generating improved bounds on some weighted cross products arising in the analysis of the delay-dependent stability problem. This class of methods is obtained by using the well-known algebraic inequality

$$
-2 \alpha^{t} \beta \leq \alpha^{t} \Xi \alpha+\beta^{t} \Xi^{-1} \beta
$$

where the vectors $\alpha, \beta \in \mathbb{R}^{n}$ and matrix $\Xi \in \mathbb{R}^{n \times n}$. An integral bounding inequality is as follows.

Lemma 20 (see [39]). Assume that $a(\alpha) \in \mathbb{R}^{n_{a}}$ and $b(\alpha) \in$ $\mathbb{R}^{n_{b}}$ are given for $\alpha \in$ Omega. Then, for any $0<X \in \mathbb{R}^{n_{a} \times n_{a}}$ and any matrix $M \in \mathbb{R}^{n_{a} \times n_{a}}$, one has

$$
-\int_{\Omega} a^{t}(\alpha) b(\alpha) d \alpha \leq \int_{\Omega}\left[\begin{array}{l}
a(\alpha) \\
b(\alpha)
\end{array}\right]^{t}
$$$$
\cdot\left[\begin{array}{cc}
X & X M \\
\bullet & \left(M^{t} X+I\right) X^{-1}\left(M^{t} X+I\right)^{t}
\end{array}\right]\left[\begin{array}{l}
a(\alpha) \\
b(\alpha)
\end{array}\right] d \alpha
$$

which when applied to time-delay systems of the type (30), it yields the following. 
Theorem 21. The time-delay system (30) is asymptotically stable for any delay satisfying $0<h \leq h_{M}$ if there exist matrices $\mathscr{P}>0, Q, \mathscr{V}$, and $\mathscr{W}$ such that

$$
\left[\begin{array}{cccc}
\Phi & -\mathscr{W}^{t} A_{d}^{t} & A_{0}^{t} A_{d}^{t} \mathscr{V} & h_{M}\left(\mathscr{P}+\mathscr{W}^{t}\right) \\
\bullet & -\mathscr{Q} & A_{d}^{t} A_{d}^{t} \mathscr{V} & 0 \\
\bullet & \bullet & -\mathscr{V} & 0 \\
\bullet & \bullet & \bullet & -\mathscr{V}
\end{array}\right]<0
$$

$\Phi$

$$
\begin{aligned}
= & \mathscr{P}\left(A_{0}+A_{d}\right)+\left(A_{0}+A_{d}\right)^{t} \mathscr{P}+\mathscr{W}^{t} A_{d} \\
& +A_{d}^{t} \mathscr{W}_{1}+\mathscr{Q} .
\end{aligned}
$$

An improved version of Lemma 20 is expressed by the following.

Lemma 22 (see [40]). Assume that $a(\alpha) \in \mathbb{R}^{n_{a}}$ and $b(\alpha) \epsilon$ $\mathbb{R}^{n_{b}}$ and $\mathcal{N}(\alpha) \in \mathbb{R}^{n_{a} \times n_{b}}$ are given for $\alpha \in$ Omega. Then, for any $0<X \in \mathbb{R}^{n_{a} \times n_{a}}$ and any matrix $M \in \mathbb{R}^{n_{a} \times n_{a}}$, one has

$$
\begin{aligned}
& -\int_{\Omega} a^{t}(\alpha) \mathcal{N}(\alpha) b(\alpha) d \alpha \\
& \quad \leq \int_{\Omega}\left[\begin{array}{l}
a(\alpha) \\
b(\alpha)
\end{array}\right]^{t}\left[\begin{array}{cc}
X & Y-\mathcal{N}(\alpha) \\
\bullet & Z
\end{array}\right]\left[\begin{array}{l}
a(\alpha) \\
b(\alpha)
\end{array}\right] d \alpha,
\end{aligned}
$$

where

$$
\left[\begin{array}{ll}
X & Y \\
\bullet & Z
\end{array}\right] \geq 0 .
$$

By considering the following LKF,

$$
\begin{aligned}
V\left(t, x_{t}\right)= & x^{t}(t) \mathscr{P} x(t)+\int_{t-d}^{t} x^{t}(\alpha) \mathcal{Q} x(\alpha) d \alpha \\
& +\int_{-d}^{0} \int_{t+\beta}^{t} \dot{x}^{t}(\alpha) Z \dot{x}(\alpha) d \alpha d \beta .
\end{aligned}
$$

Applying Lemma 22, we obtain the following delay-dependent stability theorem.

Theorem 23. The time-delay system (30) is asymptotically stable for any delay satisfying $0<d \leq d_{M}$ if there exist matrices $\mathscr{P}>0, \mathcal{Q}, X, Y$, and $Z$ such that

$$
\begin{aligned}
& {\left[\begin{array}{ccc}
\Lambda & \mathscr{P} A_{d}-Y & d_{M} A_{0}^{t} Z \\
\bullet & -Q & d_{M} A_{d}^{t} Z \\
\bullet & \bullet & -d_{M} Z
\end{array}\right]<0,} \\
& {\left[\begin{array}{cc}
X & Y \\
\bullet & Z
\end{array}\right] \geq 0,} \\
& \quad=\mathscr{P}\left(A_{0}+A_{d}\right)+\left(A_{0}+A_{d}\right)^{t} \mathscr{P}+\mathscr{W}^{t} A_{d} \\
& \quad+A_{d}^{t} \mathscr{W}_{1}+\mathscr{Q} .
\end{aligned}
$$

On the other hand, deploying Lemma 1 together with Lemma 22, a different delay-dependent stability criterion is provided by the following theorem.

Theorem 24 (see [41]). The time-delay system (30) is asymptotically stable for any delay satisfying $0<d \leq d_{M}$ if there exist matrices $\mathscr{P}_{1}>0, \mathcal{S}, P_{2}, P_{3}, P_{4}, Y_{1}, Y_{2}, Z_{1}, Z_{2}, Z_{3}$ and $R>0$ such that the following LMIs hold:

$$
\begin{aligned}
{\left[\begin{array}{ccc}
\Theta_{1} & \Theta_{2} & \Theta_{3} \\
\bullet & \Theta_{4} & \Theta_{5} \\
\bullet & \bullet & \Theta_{6}
\end{array}\right]<0 } \\
{\left[\begin{array}{ccc}
R & Y_{1} & Y_{2} \\
\bullet & Z_{1} & Z_{2} \\
\bullet & \bullet & Z_{3}
\end{array}\right] \geq 0 } \\
\Theta_{1}=A_{0}^{t} P_{2}+P_{2}^{t} A_{0}+Y_{1}+Y_{1}^{t}+\mathcal{S} \\
\quad+d_{M} Z_{1}, \\
\Theta_{2}=\mathscr{P}_{1}-P_{2}^{t}+A_{0}^{t} P_{3}+Y_{2}+d_{M} Z_{2}, \\
\Theta_{3}=P_{2}^{t} A_{d}-Y_{1}^{t}+A_{0}^{t} P_{4}, \\
\Theta_{4}=d_{M}\left(R+Z_{3}\right)-P_{3}^{t}-P_{3}, \\
\Theta_{5}=P_{3}^{t} A_{d}-Y_{2}^{t}-P_{4}, \\
\Theta_{6}=A_{d}^{t} P_{4}+P_{4}^{t} A_{d}-\mathcal{S} .
\end{aligned}
$$

Remark 25. The inequality in Lemma 22 is more general than both inequalities (59) and (60) and for this reason, it was extensively used in dealing with various issues related to timedelay systems to obtain delay-dependent results.

Now, we present another important inequality, which is also effective in the derivation of DDS conditions.

Lemma 26 (see [42]). For any constant matrix $0<\mathscr{M} \in$ $\mathbb{R}^{m \times m}$, scalars $b>a$, and vector function $\omega:[a, b] \rightarrow \mathbb{R}^{m}$ such that the integrations in the following are well-defined, then

$$
\begin{aligned}
& (b-a) \int_{a}^{b} \omega^{t}(\alpha) \mathscr{M} \omega(\alpha) d \alpha \\
& \geq \int_{a}^{b}\left[\int_{a}^{b} \omega^{t}(\alpha) d \alpha\right]^{t} \mathscr{M}\left[\int_{a}^{b} \omega^{t}(\alpha) d \alpha\right] .
\end{aligned}
$$

Using Lemma 26 and selecting the LKF

$$
\begin{aligned}
& V\left(t, x_{t}\right)= x^{t}(t) \mathscr{P} x(t)+\int_{t-d}^{t} x^{t}(\theta) \mathscr{Q} x(\theta) d \theta \\
&+d \int_{-d}^{0} \int_{t+\alpha}^{t} \dot{x}^{t}(\theta) \mathscr{X} \dot{x}(\theta) d \theta d \alpha, \\
& \mathscr{P}>0, \mathbb{Q}>0, \mathscr{Z}>0,
\end{aligned}
$$

we obtain the following stability result. 
Theorem 27 (see [6]). The time-delay system (30) is asymptotically stable for any delay satisfying $0<d \leq d_{M}$ if there exist matrices $\mathscr{P}>0, \mathbb{Q}>0$ and $\mathscr{Z}>0$ such that

$$
\left[\begin{array}{ccc}
\mathscr{P} A_{0}+A_{0}^{t} \mathscr{P}+Q-\mathscr{X} & \mathscr{P} A_{d}+\mathscr{Z} & d_{M} A_{0}^{t} \mathscr{Z} \\
\bullet & -Q \mathcal{Q}-\mathscr{P} & d_{M} A_{d}^{t} \mathscr{Z} \\
\bullet & \bullet & -\mathscr{Z}
\end{array}\right]<0 .
$$

Alternatively, selecting the LKF

$$
\begin{aligned}
& \mathbb{V}\left(t, x_{t}\right)= x^{t}(t) \mathscr{P} x(t) \\
&+ \tau_{M} \int_{-\tau_{M}}^{0} \int_{t+\alpha}^{t} \dot{x}^{t}(\theta) \mathscr{Z} \dot{x}(\theta) d \theta d \alpha, \\
& \mathscr{P}>0, \mathscr{Z}>0,
\end{aligned}
$$

we obtain the following stability result.

Theorem 28 (see [6]). The time-delay system (37) is asymptotically stable for all continuous delay $\tau(t)$ satisfying $0<\tau(t) \leq$ $\tau_{M}$ if there exist matrices $\mathscr{P}>0$ and $\mathscr{Z}>0$ such that

$$
\left[\begin{array}{ccc}
\mathscr{P} A_{0}+A_{0}^{t} \mathscr{P}-\mathscr{Z} & \mathscr{P} A_{d}+\mathscr{Z} & \tau_{M} A_{0}^{t} \mathscr{Z} \\
\bullet & -\mathscr{Z}-\mathscr{P} & \tau_{M} A_{d}^{t} \mathscr{Z} \\
\bullet & \bullet & -\mathscr{Z}
\end{array}\right]<0 .
$$

A useful result is summarized by the following lemma.

Lemma 29 (the integral inequality [43]). For any constant matrix $0<\Sigma \in \mathbb{R}^{n \times n}$, scalar $\tau_{*}<\tau(t)<\tau^{+}$, and vector function $\dot{x}:\left[t-\tau^{+}, t-\tau_{*}\right] \rightarrow \mathbb{R}^{n}$ such that the following integration is well-defined, then it holds that

$$
\begin{gathered}
-\left(\tau^{+}-\tau_{*}\right) \int_{t-\tau^{+}}^{t-\tau_{*}} \dot{x}^{t}(s) \sum \dot{x}(s) d s \\
\leq-\left[x\left(t-\tau_{*}\right)-x\left(t-\tau^{+}\right)\right]^{t} \\
\cdot \Sigma\left[x\left(t-\tau_{*}\right)-x\left(t-\tau^{+}\right)\right] .
\end{gathered}
$$

Lemma 29 is frequently called the "integral inequality" and it is derived from Jensen's inequality [44].

Remark 30. It is significant to observe that Theorem 27 establishes that the time-delay system (30) is asymptotically stable for any delay $d$ satisfying $0<d \leq d_{M}$ when the LMI (69) attains a feasible solution, which implies that, for $d$ satisfying $0<d \leq d_{M} / 2$, the time-delay system (30) is asymptotically stable as well. Then, introducing the half delay into the time-delay system (30) will take more information on the system and thus may tend to reduce the conservatism in Theorem 27. For further elaboration on this argument, see [45].

6.7. Discrete-Time Systems. Less attention has been paid to discrete-time systems with a time-delay because a linear discrete-time system with a constant time-delay can be transformed into a delay-free system by means of a state-augmentation approach. However this approach is not suitable for systems with either unknown or time-varying delays. For a small time-varying delays, the descriptor model transformation approach was employed [46].

Consider a class of discrete-time systems with state-delay is represented by

$$
x(k+1)=A_{o} x(k)+D_{o} x(k-d(k))
$$

where for $k \in Z_{+} \triangleq\{0,1, \ldots\}, x(k) \in \mathfrak{R}^{n}$ is the state and $A_{o} \in \mathfrak{R}^{n \times n}$ and $D_{o} \in \mathfrak{R}^{n \times n}$ are constant matrices. The delay factor $d(k)$ is unknown-but-bounded in the form

$$
0<d_{m} \leq d(k) \leq d_{M}, \quad d_{s}=d_{M}-d_{m}+1,
$$

where the scalars $d_{m}$ and $d_{M}$ represent the lower and upper bounds, respectively, and $d_{s}$ denotes the number of samples within the delay interval.

Remark 31. By setting $d(k) \equiv 0$ in (73), it is readily seen that $\left|\lambda\left(A_{o}+D_{o}\right)\right|<1$ is a necessary condition for stability of system (73). From all studies on discrete-time-delay systems, it is assumed that this is always the case.

Remark 32. The class of systems (73) represents a nominally linear model which emerges in many areas dealing with the applications functional difference equations or delaydifference equations. These applications include cold rolling mills, decision-making processes, and manufacturing systems.

Related results for a class of discrete-time systems with time-varying delays can be found in [47] where delaydependent stability and stabilization conditions were derived. It should be stressed that although we consider only the case of single time-delay, extension to multiple time-delay systems can be easily attained using an augmentation procedure.

Intuitively if we associate with system (73) a positivedefinite Lyapunov-Krasovskii functional $V(k, x(k))>0$ and we find that its first difference $\Delta V(k, x(k))=V(k+1, x(k+$ $1))-V(k, x(k))$ is negative-definite along the solutions of (73), then the origin of system (73) is globally asymptotically stable. Formally, we present the following theorem for discrete-time systems of the type (73).

Theorem 33. The equilibrium $\mathbf{0}$ of the discrete-time system

$$
x(k+1)=h(x(k))
$$

is globally asymptotically stable if there is a function $V$ : $\{0,1,2, \ldots\} \times \mathfrak{R}^{n} \rightarrow \mathfrak{R}$ such that

(i) $V(k, x(k))$ is a positive-definite function, decrescent, and radially unbounded,

(ii) $\Delta V(k, x(k))=V(k+1, x(k+1))-V(k, x(k))$ is negative-definite along the solutions of system (73).

For arbitrary value of $d(k)$, denote

$$
z(k)=\left[x^{t}(k) \mid x^{t}(k-d(k))\right]^{t} .
$$


We have

$$
z(k+1)=\left[\begin{array}{ccccc}
A_{o} & 0 & \ldots & 0 & D_{o} \\
I & 0 & \ldots & 0 & 0 \\
\vdots & \ddots & \ldots & \vdots & \vdots \\
0 & 0 & \ldots & I & 0
\end{array}\right] z(k)
$$

It is obvious that system (73) is globally asymptotically stable if and only if system (75) is globally asymptotically stable. For system (75), we define

$$
\widehat{V}(k, z(k))=z^{t}(k) \operatorname{diag}\left[\begin{array}{llll}
\mathscr{P} & Q & \cdots & Q
\end{array}\right] z(k),
$$

where $\mathscr{P}>0$ and $Q \mathcal{Q}$. It is easy to see that $\widehat{V}(k, z(k))>0$, decrescent and radially unbounded, and hence system (75) is globally asymptotically stable.

By selecting the Lyapunov-Krasovskii functional

$$
\begin{aligned}
& V(k)=x^{t}(k) \mathscr{P} x(k)+\sum_{m=k-d(k)}^{k-1} x^{t}(m) \mathscr{Q} x(m), \\
& 0<\mathscr{P}, 0<\mathbb{Q},
\end{aligned}
$$

and invoking the Lyapunov-Krasovskii theorem, the following stability condition can be derived.

Theorem 34. The discrete-delay system (73) is asymptotically stable if there exist matrices $\mathscr{P}>0$ and $\mathbb{Q}>0$ such that

$$
\left[\begin{array}{ccc}
-(\mathscr{P}-\mathscr{Q}) & 0 & A_{o}^{t} \mathscr{P} \\
\bullet & -Q \mathcal{Q} & D_{o}^{t} \mathscr{P} \\
\bullet & \bullet & -\mathscr{P}
\end{array}\right]<0 .
$$

We stress that LMI (80) is virtually delay-independent since it is satisfied no matter the size of delay $d(k)$ is.

Next, sufficient delay-dependent LMI-based stability conditions are given. The approach used here does not introduce any dynamics and leads to a product separation between the matrices of the system and those from the LyapunovKrasovskii functional. The following theorem provides some LMI conditions depending on the values $d_{m}$ and $d_{M}$.

Theorem 35. Given the delay sample number $d_{s}$, system (73) subject to (74) is delay-dependent asymptotically stable if one of the following equivalent conditions is satisfied:

(A) There exist matrices $0<\mathscr{P} \in \mathfrak{R}^{n \times n}$ and $0<Q \in \mathfrak{R}^{n \times n}$ such that

$$
\Xi_{a}=\left[\begin{array}{cc}
A_{o}^{t} \mathscr{P} A_{o}+d_{s} Q-\mathscr{P} & A_{o}^{t} \mathscr{P} D_{o}^{t} \\
\bullet & D_{o}^{t} \mathscr{P} D_{o}-Q
\end{array}\right]<0 .
$$

(B) There exist matrices $0<\mathscr{P} \in \mathfrak{R}^{n \times n}, 0<Q \in \mathfrak{R}^{n \times n}$, $\mathscr{X} \in \mathfrak{R}^{n \times n}, \mathscr{Y} \in \mathfrak{R}^{n \times n}$, and $\mathscr{Z} \in \mathfrak{R}^{n \times n}$ such that

$$
\begin{aligned}
\Xi_{c} & =\left[\begin{array}{ccc}
\mathscr{P}+\mathscr{X}+\mathscr{X}^{t} & \mathscr{Y}-\mathscr{X} A_{o} & \mathscr{Z}-\mathscr{X} D_{o} \\
\bullet & \Gamma_{v} & -A_{o}^{t} \mathscr{X}^{t}-\mathscr{Y} D_{o} \\
\bullet & \bullet & \Gamma_{w}
\end{array}\right] \\
& <0
\end{aligned}
$$

where

$$
\begin{aligned}
& \Gamma_{v}=-A_{o}^{t} \mathscr{Z}^{t}-\mathscr{Y} A_{o}+d_{s} \mathscr{Q}-\mathscr{P}, \\
& \Gamma_{v}=-\mathscr{Q}-\mathscr{Z} D_{o}-D_{o}^{t} \mathscr{Z}^{t} .
\end{aligned}
$$

In this case, the Lyapunov-Krasovskii functional (LKF)

$$
\begin{aligned}
\widetilde{V}(k)= & x^{t}(k) \mathscr{P} x(k)+\sum_{m=k-d(k)}^{k-1} x^{t}(m) \mathscr{Q} x(m) \\
& +\sum_{s=2-d_{M}}^{1-d_{m}} \sum_{m=k+s-1}^{k-1} x^{t}(m) \mathscr{Q} x(m)>0
\end{aligned}
$$

is such that

$$
\Delta \widetilde{V}(k)<0, \quad \forall\left[x^{t}(k) x^{t}(k-d(k))\right]^{t} \neq \mathbf{0} .
$$

The result of Theorem 35 was developed in [47-49].

Next, we consider the following discrete-time piecewise linear systems with infinite distributed delays [50]:

$$
x(k+1)=A_{\ell} x(k)+D_{\ell} \sum_{d=1}^{\infty} \mu_{d} x(k-d)+B_{\ell} u(k),
$$

where $x(k) \in \mathfrak{R}^{n}$ is the state and $\left\{S_{\ell}\right\}_{\ell \in L}$ denotes a partition of the state-space into a number of closed polyhedral subspaces, $L$ is the index set of subspaces, and $u(k) \in \mathfrak{R}^{m}$ is the control input. Matrices $A_{\ell} D_{\ell} B_{\ell}$ are constant matrices with appropriate dimensions corresponding to the $\ell$ th local model of the systems. When the state of the system transits from one region to another at the time $k$, the dynamics is governed by the local model of the former one. $\mu_{d} \geq 0$ is the convergence constants that satisfy the following condition:

$$
\bar{\mu} \triangleq \sum_{d=1}^{\infty} \mu_{d} \leq \sum_{d=1}^{\infty} d \mu_{d}<+\infty .
$$

Distributed time-delays have been widely recognized and intensively studied for continuous-time systems [51]. However, the corresponding results for discrete-time systems have been very few due mainly to the difficulty in formulating the distributed delays in a discrete-time domain. The distributed delay term $\sum_{d=1}^{\infty} \mu_{d} x(k-d)$ can be regarded as the discretization of the infinite integral form $\int_{-\infty}^{t} k(t-s) x(s) d s$ for the continuous-time system. The following result is recalled [51].

Lemma 36. Let $0 \leq M \in \mathfrak{R}^{n \times n}, x_{j} \in \mathfrak{R}^{n}$, and $a_{j}>0$, $j=1,2, \ldots$, are constants. If the series concerned is convergent, then one has

$$
\left(\sum_{j=1}^{\infty} a_{j} x_{j}\right)^{t} M\left(\sum_{j=1}^{\infty} a_{j} x_{j}\right) \leq\left(\sum_{j=1}^{\infty} a_{j}\right) \sum_{j=1}^{\infty} a_{j} x_{j}^{t} M x_{j} .
$$

Introduce the following Lyapunov-Krasovskii functional candidate:

$$
V(k)=x^{t}(k) \mathscr{P}_{\ell} x(k)+\sum_{d=1}^{\infty} \mu_{d} \sum_{m=k-d}^{k-1} x^{t}(m) \mathscr{Q} x(m) .
$$


By setting $\bar{Q}=\overline{\mu_{d}} \mathscr{Q}$ and invoking Theorem 34, the following result is obtained.

Theorem 37. Consider the piecewise linear system (86) with $u \equiv 0$. If there exist matrices $X_{\ell}>0$ and $\bar{Q}>0$ such that the following linear matrix inequalities hold for $(\ell, j) \in \Omega \triangleq\{\ell, j \mid$ $\left.x(k) \in S_{\ell}, x(k+1) \in S_{j}, j \neq \ell\right\}:$

$$
\left[\begin{array}{ccc}
-\mathscr{P}+\bar{Q} & 0 & A_{o}^{t} \mathscr{P} \\
\bullet & -\frac{\bar{Q}}{\overline{\mu_{d}}} & D_{o}^{t} \mathscr{P} \\
\bullet & \bullet & -\mathscr{P}
\end{array}\right]<0 .
$$

We emphasize that Theorem 37 was established in [50].

\section{Model Transformations}

It must be recalled that the prototype system (30), the independent of delay (IOD) stability demands matrix $A_{0}$ to be Hurwitz which, coherently, can be found in condition (33). On the other hand, the criteria ensuring delay-dependent stability for $h \in\left[0, h_{M}\right)$ require the matrix $A_{0}+A_{d}$ to be Hurwitz as evident in condition (36). On this basis, several results concerning delay-dependent stability were derived, from the formula

$$
\int_{t-d}^{t} \dot{x}(s) d s=x(t)-x(t-d) .
$$

Consider the change of variables

$$
\begin{aligned}
A_{j} x(t-d)= & {\left[A_{j}-L_{j}\right] x\left(t-d_{j}\right) } \\
& +L_{j}\left[x(t)-\int_{t-d_{j}}^{t} \dot{x}(s) d s\right] .
\end{aligned}
$$

This will transform the multiple-delay system with possibly $d_{1}=0$

$$
\dot{x}(t)=\sum_{j=1}^{m} A_{j} x\left(t-d_{j}\right)
$$

into the system having augmented delay $h=\max \left(h_{j}+h_{k}\right)$

$$
\begin{aligned}
\dot{x}(t)= & {\left[\sum_{j=1}^{m} L_{j}\right] x(t)+\sum_{j=1}^{m} A_{j} x\left(t-d_{j}\right) } \\
& +\sum_{j=1, k=1}^{m} \int_{t-d_{j}}^{t} L_{j} A_{k} x\left(s-d_{j}\right) d s .
\end{aligned}
$$

Model (94) guarantees that the unstable nondelayed part $A_{d}$ in system (30) is absorbed in the stable part $\left[\sum_{j=1}^{m} L_{j}\right]$. Indeed such decomposition can be conveniently handled using LMI tools. It is shown in $[5,52]$ that the foregoing system can be written in the three following forms:

$$
\begin{aligned}
& \dot{x}(t)=A x(t)-\sum_{j, k=1}^{m} A_{j k} \int_{t-d_{j k}}^{t-d_{k}} x(s) d s, \\
& \dot{x}(t)=A x(t)-\sum_{j=1}^{m} A_{j} \int_{t-d_{j}}^{t} \dot{x}(s) d s, \\
& \frac{d}{d t}\left[x(t)+\sum_{j=1}^{m} A_{j} \int_{t-d_{j}}^{t} x(s) d s\right]=A x(t), \\
& A=\sum_{j=1}^{m} A_{j}, A_{j k}=A_{j} A_{k}, d_{j k}=d_{j}+d_{k} .
\end{aligned}
$$

It turns out that each of the above formulations can be studied by using specific Lyapunov-Krasovskii functionals (34) leading to the three different Riccati equations [5]:

$$
\begin{aligned}
& \Pi+m d \mathscr{R}+\mathscr{P} \sum_{j, k=1}^{m} d_{j} A_{j k} \mathscr{R}^{-1} B_{j k}^{t} \mathscr{P}=-\mathscr{Q}, \\
& \Pi+\sum_{j=1}^{m}\left(d_{j} \mathscr{P} A_{j} \mathscr{R}^{-1} B_{j}^{t} \mathscr{P}+m d A_{j}^{t} \mathscr{R} A_{j}\right)=-\mathscr{Q}, \\
& \Pi+\sum_{j=1}^{m} d_{j} \mathscr{R}_{j}+\sum_{j, k=1}^{m} d_{j} A^{t} \mathscr{P} A_{j} \mathscr{R}_{j}^{-1} A_{j}^{t} \mathscr{P} A=-\mathscr{Q}, \\
& \Pi=\mathscr{P} A+A^{t} \mathscr{P} .
\end{aligned}
$$

Remark 38. In the literature, there were other different methods to develop delay-dependent stability criteria. These methods include the discretized LKF approach [4], the descriptor system approach [53], and the delay-partitioning projection approach [54]. Declaring the stability result as conservative or not requires well-defined quantitative measures. More importantly, it must be pointed out that the issue of computational complexity and the associated number of manipulated matrices deserve a serious investigation.

\section{Delay-Dependent Stabilization}

Extending the time-delay system (30) for stabilization studies, we start with the form

$$
\dot{x}(t)=A_{0} x(t)+A_{d} x(t-d)+B_{0} u(t), \quad t \geq t_{0},
$$

where $u(t)$ is the control input and $B_{0}$ is the input matrix with the pair $A_{0}, B_{0}$ being controllable. We seek to design a state feedback controller

$$
u(t)=\mathbb{K}_{0} x(t)
$$

such that the closed-loop system

$$
\dot{x}(t)=A_{c} x(t)+A_{d} x(t-d), \quad A_{c}=A_{0}+B_{0} \mathbb{K}_{0},
$$

is asymptotically stable [55]. This is attained by convex analysis [31] leading to the following theorem. 
Theorem 39. The closed-loop time-delay system (97) is asymptotically stable for any $d \geq 0$ if there exist matrices $\mathscr{X}>$ $0, \mathscr{Y}, \mathscr{W}>0$ verifying

$$
\left[\begin{array}{cc}
A_{0} \mathscr{X}+\mathscr{X} A_{0}^{t}+B_{0} \mathscr{Y}+\mathscr{Y}^{t} B_{0}^{t}+\mathscr{W} & A_{d} \mathscr{X} \\
\bullet & -\mathscr{W}
\end{array}\right]<0 .
$$

8.1. A Class of Nonlinear Systems. One of the standard classes of nonlinear time-delay systems is given by

$$
\begin{aligned}
\dot{x}(\sigma)= & A_{o} x(\sigma)+A_{d} x\left(\sigma-\sigma_{d}\right)+B_{o} u(\sigma) \\
& +h(t, x, x(t-d))
\end{aligned}
$$

in the dimensionless coordinates $\sigma$, where $x(\sigma)$ is the state vector, $u(t)$ is the control input, and $A_{o}, B_{o}$, and $A_{d}$ are known real constant matrices. The nonlinear vector function $h(\cdot, \cdot)$ is a piecewise-continuous function in its arguments. In the discussions to follow, we assume that this function is uncertain satisfying the quadratic inequality

$$
\begin{aligned}
& h^{t}(t, x, x(t-d)) h(t, x, x(t-d)) \\
& \quad \leq \alpha^{2} x^{t} H_{o}^{t} H_{o} x+\theta^{2} x^{t}(t-d) H_{d}^{t} H_{d} x(t-d),
\end{aligned}
$$

where $\alpha>0$ and $\theta>0$ are the bounding parameters. The matrices $H_{o} \in \mathbb{R}^{r \times n}$ and $H_{d} \in \mathbb{R}^{p \times n}$ are constants and characterize the upper bound on system nonlinearities.

For stability purposes, we let $\alpha^{-2}=\gamma$ and $\theta^{-2}=\psi$. The following convex optimization result holds.
Theorem 40. Nonlinear system (101) with $u \equiv 0$ is robustly stable if the following LMI feasibility problem is solvable:

$$
\begin{aligned}
\min & \gamma, \psi \\
\text { subject to } & \mathscr{X}>0 \\
& {\left[\begin{array}{ccccccc}
A_{o} \mathscr{X}+\mathscr{X} A_{o}^{t} & \mathscr{X} \mathscr{W} & \mathscr{X} H_{o}^{t} & I & A_{d} & 0 \\
\bullet & -\mathscr{W} & 0 & 0 & 0 & 0 \\
\bullet & \bullet & -\gamma I & 0 & 0 & 0 \\
\bullet & \bullet & \bullet & -I & 0 & 0 \\
\bullet & \bullet & \bullet & \bullet & -W & H_{d}^{t} \\
\bullet & \bullet & \bullet & \bullet & \bullet & -\psi
\end{array}\right] } \\
& <0 .
\end{aligned}
$$

Given that the pair $\left(A_{o}, B_{o}\right)$ is stabilizable. We achieve state feedback stabilization in two stages as follows.

(S1) Let the linear state feedback be $u(t)=K_{o} x(t)$, and then the closed-loop system becomes

$$
\begin{array}{r}
\dot{x}(t)=A_{k} x(t)+A_{d} x(t-d)+h(t, x, x(t-d)), \\
A_{k}=\left(A_{o}+B_{o} K_{o}\right) .
\end{array}
$$

This establishes the following theorem.

Theorem 41. Nonlinear system (101) is robustly stabilized by control law $u(t)=K_{o} x(t)$, if the following LMI problem has a feasible solution.

$$
\begin{aligned}
& \min \gamma, \psi \\
& \text { subject to } \mathscr{X}>0 \text {, } \\
& y \\
& {\left[\begin{array}{cccccc}
A_{o} \mathscr{X}+\mathscr{X} A_{o}^{t}+B_{o} \mathscr{Y}+\mathscr{Y}^{t} B_{o}^{t} & \mathscr{X} \mathscr{W} & \mathscr{X} H_{o}^{t} & I & A_{d} & 0 \\
\bullet & -\mathscr{W} & 0 & 0 & 0 & 0 \\
\bullet & \bullet & -\gamma I & 0 & 0 & 0 \\
\bullet & \bullet & \bullet & -I & 0 & 0 \\
\bullet & \bullet & \bullet & \bullet & -W & H_{d}^{t} \\
\bullet & \bullet & \bullet & \bullet & \bullet & -\psi
\end{array}\right]<0 .}
\end{aligned}
$$

(S2) Next, to include bounds the gain matrix $K_{o}$, we set the bounding relations

$$
\begin{aligned}
M^{t} M & <\mu I, \quad \mu>0, \\
X^{-1} & <\varphi I, \quad \varphi>0 .
\end{aligned}
$$

Moreover, to guarantee desired values $\{\bar{\alpha}, \bar{\theta}\}$ of the bounding factors $\{\alpha, \theta\}$, we enforce $\alpha^{-2}=\gamma$ and $\theta^{-2}=\psi$. The following theorem summarizes the main result.

Theorem 42. Nonlinear system (101) is robustly stabilized by control law $u(t)=K_{o} x(t)$, with constrained feedback gains if 
following convex optimization problem over LMIs has a feasible solution:

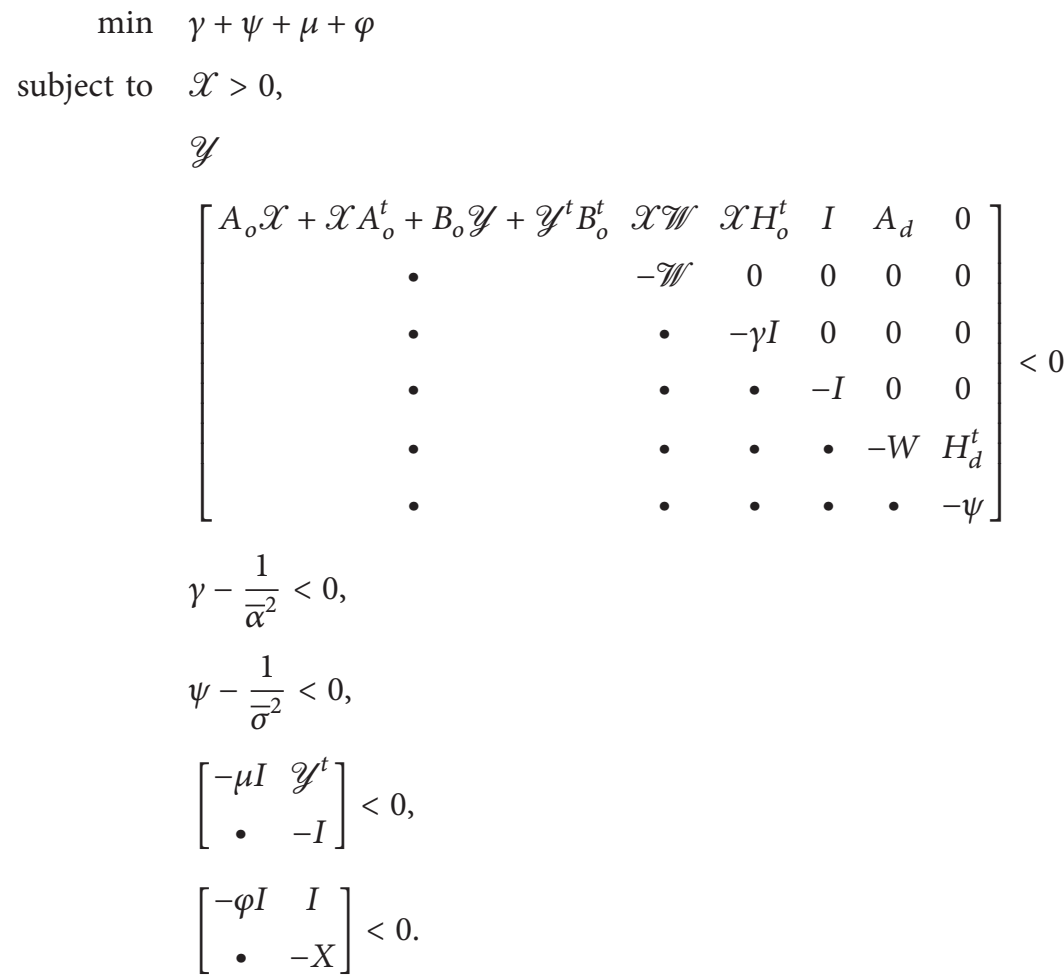

Remark 43. One can address the performance deterioration issue by considering that the actual linear state feedback controller has the form $u(t)=\left[K_{o}+\Delta K_{o}\right] x(t), K_{o} \in \mathbb{R}^{m \times n}$ is a constant gain matrix, and $\Delta K_{o}$ is a gain perturbation matrix.

\section{Kalman Filtering}

The seminal Kalman filtering algorithm [56] is the optimal estimator over all possible linear ones and gives unbiased estimates of the unknown state vectors under the conditions that the system and measurement noise processes are mutually independent Gaussian distributions. Robust stateestimation arose out of the desire to estimate unmeasurable state variables when the plant model has uncertain parameters. In the sequel, we consider the state-estimation problem for a class of linear continuous-time-lag systems with normbounded parameter uncertainties. Specifically, we address the state-estimator design problem such that the estimation error covariance has a guaranteed bound for all admissible uncertainties.

9.1. A Class of Continuous-Time-Lag Systems. We consider a class of uncertain time-delay systems represented by

$$
\begin{aligned}
\dot{x}(t)= & {[A(t)+\Delta A(t)] x(t)+A_{d}(t) x(t-\tau) } \\
& +w(t) \\
= & A_{\Delta}(t) x(t)+A_{d}(t) x(t-\tau)+w(t),
\end{aligned}
$$

$$
\begin{aligned}
y(t) & =[C(t)+\Delta C(t)] x(t)+v(t) \\
& =C_{\Delta}(t) x(t)+v(t)
\end{aligned}
$$

where $x(t) \in \mathbb{R}^{n}$ is the state, $y(t) \in \mathbb{R}^{m}$ is the measured output, and $w(t) \in \mathbb{R}^{n}$ and $v(t) \in \mathbb{R}^{m}$ are, respectively, the process and measurement noises. In (1)-(2), $A(t) \in \mathbb{R}^{n \times n}$, $A_{d}(t) \in \mathbb{R}^{n \times n}$, and $C(t) \in \mathbb{R}^{m \times n}$ are piecewise-continuous matrix functions. Here, $\tau$ is a constant scalar representing the amount of time-lag in the state. The matrices $\Delta A(t)$ and $\Delta C(t)$ represent time-varying parametric uncertainties which are of the form:

$$
\left[\begin{array}{l}
\Delta A(t) \\
\Delta C(t)
\end{array}\right]=\left[\begin{array}{l}
H(t) \\
H_{c}(t)
\end{array}\right] \Delta(t) E(t)
$$

where $H(t) \in \mathbb{R}^{n \times \alpha}, H_{c}(t) \in \mathbb{R}^{m \times \alpha}$, and $E(t) \in \mathbb{R}^{\beta \times n}$ are known piecewise-continuous matrix functions and $\Delta(t) \epsilon$ $\mathbb{R}^{\alpha \times \beta}$ is an unknown matrix with Lebesgue measurable elements satisfying

$$
\Delta^{t}(t) \Delta(t) \leq I \quad \forall t
$$

The initial condition is specified as $\langle x(0), x(s)\rangle=\left\langle x_{o}, \phi(s)\right\rangle$, where $\phi(\cdot) \in \mathscr{L}_{2}[-\tau, 0]$ which is assumed to be a zero-mean Gaussian random vector. The following standard assumptions on noise statistics are recalled. 
Assumption 44. $\forall t, s \geq 0$

(a)

$$
\begin{aligned}
\mathbb{E}[w(t)] & =0 ; \\
\mathbb{E}\left[w(t) w^{t}(s)\right] & =W(t) \delta(t-s) ;
\end{aligned}
$$

$W(t)>0$.

(b)

$$
\begin{aligned}
\mathbb{E}[v(t)] & =0 ; \\
\mathbb{E}\left[v(t) v^{t}(s)\right] & =V(t) \delta(t-s) ;
\end{aligned}
$$

$$
V(t)>0 .
$$

(c)

$$
\begin{aligned}
& \mathbb{E}\left[x(0) w^{t}(t)\right]=0 ; \\
& \mathbb{E}\left[x(0) v^{t}(t)\right]=0 .
\end{aligned}
$$

(d)

$$
\begin{aligned}
\mathbb{E}\left[w(t) v^{t}(s)\right] & =0 ; \\
\mathbb{E}\left[x(0) x^{t}(0)\right] & =R_{o},
\end{aligned}
$$

where $\mathbb{E}[\cdot]$ stands for the mathematical expectation and $\delta(\cdot)$ is the Dirac function.

9.2. Robust Kalman Filtering. Our objective is to design a stable state estimator of the form:

$$
\dot{\hat{x}}(t)=G(t) \widehat{x}(t)+K(t) y(t), \quad \widehat{x}(0)=0,
$$

where $G(t) \in \mathbb{R}^{n \times n}$ and $K(t) \in \mathbb{R}^{n \times m}$ are piecewisecontinuous matrices to be determined such that there exists a matrix $\Psi \geq 0$ satisfying

$$
\mathbb{E}\left[(x-\widehat{x})(x-\widehat{x})^{t}\right] \leq \Psi, \quad \forall \Delta: \Delta^{t}(t) \Delta(t) \leq I .
$$

Note that (112) implies

$$
\mathbb{E}\left[(x-\widehat{x})^{t}(x-\widehat{x})\right] \leq \operatorname{tr}(\Psi), \quad \forall \Delta: \Delta^{t}(t) \Delta(t) \leq I .
$$

In this case, the estimator (116) is said to provide a guaranteed cost (GC) matrix $\Psi$.

Examination of the proposed estimator proceeds by analyzing the estimation error

$$
e(t)=x(t)-\widehat{x}(t) .
$$

Substituting (109) and (116) into (119), we express the dynamics of the error in the form

$$
\begin{aligned}
\dot{e}(t)= & G(t) e(t)+[A(t)-G(t)-K(t) C(t)] x(t) \\
& +[\Delta A(t)-K(t) \Delta C(t)] x(t) \\
& +A_{d}(t) x(t-\tau)+[w(t)-K(t) v(t)] .
\end{aligned}
$$

By introducing the extended state vector

$$
\xi(t)=\left[\begin{array}{l}
x(t) \\
e(t)
\end{array}\right] \in \mathbb{R}^{2 n},
$$

it follows from (108)-(109) and (120) that

$$
\begin{aligned}
\dot{\xi}(t)= & {[\widehat{A}(t)+\widehat{H}(t) F(t) \widehat{E}(t)] \xi(t)+\widehat{D}(t) \xi(t-\tau) } \\
& +\widehat{B}(t) \eta(t) \\
= & \widehat{A}_{\Delta}(t) \xi(t)+\widehat{D}(t) \xi(t-\tau)+\widehat{B}(t) \eta(t),
\end{aligned}
$$

where $\eta(t)$ is a stationary zero-mean noise signal with identity covariance matrix and

$$
\widehat{A}(t)=\left[\begin{array}{cc}
A(t) & 0 \\
A(t)-G(t)-K(t) C(t) & G(t)
\end{array}\right],
$$

$$
\begin{aligned}
\widehat{H}(t) & =\left[\begin{array}{c}
H(t) \\
H(t)-K(t) H_{c}(t)
\end{array}\right], \\
\widehat{E}(t) & =\left[\begin{array}{ll}
E(t) & 0
\end{array}\right],
\end{aligned}
$$

$$
\widehat{B} \widehat{B}^{t}(t)=\left[\begin{array}{cc}
W(t) & W(t) \\
W(t) & W(t)+K(t) V(t) K^{t}(t)
\end{array}\right],
$$

$$
\begin{aligned}
\widehat{D}(t) & =\left[\begin{array}{ll}
A_{d}(t) & 0 \\
A_{d}(t) & 0
\end{array}\right], \\
\eta & =\left[\begin{array}{l}
w(t) \\
v(t)
\end{array}\right] .
\end{aligned}
$$

Definition 45. Estimator (111) is said to be a quadratic estimator (QE) associated with a matrix $\Omega(t)>0$ for system (108) if there exists a scalar $\lambda(t)>0$ and a matrix

$$
0<\Omega(t)=\left[\begin{array}{ll}
\Omega_{1}(t) & \Omega_{3}(t) \\
\Omega_{3}^{t}(t) & \Omega_{2}(t)
\end{array}\right]
$$

satisfying the algebraic inequality

$$
\begin{aligned}
& -\dot{\Omega}(t)+\widehat{A}_{\Delta}(t) \Omega(t)+\Omega(t) \widehat{A}_{\Delta}^{t}(t)+\lambda(t) \Omega(t-\tau) \\
& \quad+\lambda^{-1}(t) \widehat{D}(t) \Omega(t-\tau) \widehat{D}^{t}(t)+\widehat{B}(t) \widehat{B}^{t}(t) \\
& \leq 0 .
\end{aligned}
$$

The next result shows that if (112) is QE for system (108)(109) with cost matrix $\Omega(t)$, then $\Omega(t)$ defines an upper bound for the filtering error covariance; that is,

$$
\mathbb{E}\left[e(t) e^{t}(t)\right] \leq \Omega_{2}(t) \quad \forall t
$$

for all admissible uncertainties satisfying (110)-(111).

Theorem 46. Consider the time-delay (108)-(109) satisfying (110)-(111) and with known initial state. Suppose there exists a solution $\Omega(t) \geq 0$ to inequality (125) for some $\lambda(t)>0$ and for all admissible uncertainties. Then the estimator (116) provides an upper bound for the filtering error covariance; that is,

$$
\mathbb{E}\left[e(t) e^{t}(t)\right] \leq \Omega_{2}(t) .
$$


We employ hereafter a Riccati equation approach to solve the robust Kalman filtering for time-delay systems. To this end, we define piecewise matrices $P(t)=P^{t}(t) \in \mathbb{R}^{n \times n}$; $L(t)=L^{t}(t) \in \mathbb{R}^{n \times n}$ as the solutions of the Riccati differential equations (RDE):

$$
\begin{aligned}
& \dot{P}(t)=A(t) P(t)+P(t) A^{t}(t)+\lambda(t) P(t-\tau) \\
& +\widehat{W}(t)+\lambda^{-1}(t) A_{d}(t) P(t-\tau) A_{d}^{t}(t)+\mu(t) \\
& \cdot P(t) E^{t}(t) E(t) P(t) ; \\
& P(t-\tau)=0 \quad \forall t \in[0, \tau], \\
& \dot{L}(t)=A(t) L(t)+L(t) A^{t}(t)+\lambda(t) L(t-\tau) \\
& \quad+\widehat{W}(t)+\lambda^{-1}(t) A_{d}(t) P(t-\tau) A_{d}^{t}(t)+\mu(t) \\
& \quad \cdot L(t) E^{t}(t) E(t) L(t) \\
& \quad-\left[L(t) C^{t}(t)+\mu^{-1}(t) H(t) H_{c}^{t}(t)\right] \widehat{V}^{-1}(t) \\
& \cdot\left[C(t) L(t)+\mu^{-1}(t) H_{c}(t) H^{t}(t)\right] ; \\
& L(t-\tau)=0 \quad \forall t \in[0, \tau],
\end{aligned}
$$

where $\lambda(t)>0$ and $\mu(t)>0 \forall t$ are scaling parameters and the matrices $\widehat{A}(t), \widehat{V}(t)$, and $\widehat{W}(t)$ are given by

$$
\begin{aligned}
\widehat{W}(t) & =W(t)+\mu^{-1}(t) H(t) H^{t}(t), \\
\widehat{V}(t) & =V(t)+\mu^{-1}(t) H_{c}(t) H_{c}^{t}(t), \\
\widehat{A}(t) & =A(t)+\delta A(t) \\
& =A(t)+\mu^{-1}(t) L^{t}(t) E^{t}(t) E(t) .
\end{aligned}
$$

Let the $(\lambda, \mu)$-parameterized estimator be expressed as

$$
\begin{aligned}
\dot{\hat{x}}(t)= & \left\{A(t)+\mu^{-1}(t) L^{t}(t) E^{t}(t) E(t)\right\} \hat{x}(t) \\
& +K(t)\{y(t)-C(t) \hat{x}(t)\}
\end{aligned}
$$

where the gain matrix $\mathbb{K}_{e}(t) \in \mathbb{R}^{n \times m}$ is to be determined. The following theorem summarizes the main result.

Theorem 47. Consider system (108)-(109) satisfying the uncertainty structure (110)-(111) with zero initial condition. Suppose the process and measurement noises satisfy Assumption 44. For some $\mu(t)>0, \lambda(t)>0$, let $P(t)=P^{t}(t)$ and $L(t)=L^{t}(t)$ be the solutions of $R D E$ (131)-(132), respectively. Then the $(\lambda, \mu)$ parametrized estimator (132) is QE estimator with GC such that

$$
\mathbb{E}\left[\{x(t)-\widehat{x}(t)\}^{t}\{x(t)-\widehat{x}(t)\}\right] \leq \operatorname{tr}[L(t)] .
$$

Moreover, the gain matrix $\mathbb{K}(t)$ is given by

$$
\mathbb{K}_{e}(t)=\left\{L(t) C^{t}(t)+\mu^{-1}(t) H(t) H_{c}^{t}(t)\right\} \widehat{V}^{-1}(t) .
$$

Further details can be found in [57].
Remark 48. Had we considered a class of uncertain timedelay systems represented by

$$
\begin{aligned}
x_{k+1} & =\left[A_{k}+\Delta A_{k}\right] x_{k}+D_{k} x_{k-\tau}+w_{k} \\
& =A_{k, \Delta} x_{k}+D_{k} x_{k-\tau}+w_{k}, \\
y_{k} & =\left[C_{k}+\Delta C_{k}\right] x_{k}+v_{k}=C_{k, \Delta} x_{k}+v_{k}, \\
z_{k} & =C_{1, k} x_{k},
\end{aligned}
$$

where $x_{k} \in \mathbb{R}^{n}$ is the state, $y_{k} \in \mathbb{R}^{m}$ is the measured output, $z_{k} \in \mathbb{R}^{p}$ is a linear combination of the state variables to be estimated, and $w_{k} \in \mathbb{R}^{r}$ and $v_{k} \in \mathbb{R}^{m}$ are, respectively, the process and measurement noise sequences, and following parallel development to the continuous-case, we would be able to generate a robust discrete-time Kalman filter.

\section{Neural Networks}

We consider a continuous-time-delayed uncertain neural network (UNN) which is described by the following nonlinear retarded functional differential equations:

$$
\begin{aligned}
\dot{y}(t)= & -\left(A_{\mathrm{o}}+\Delta A_{o}\right) y(t) \\
& +\left(W_{o}+\Delta W_{o}\right) g(y(t), t) \\
& +\left(W_{1}+\Delta W_{1}\right) g(y(t-\tau), t)+b,
\end{aligned}
$$

where $y(t)=\left[y_{1}(t), \ldots, y_{n}(t)\right]^{t} \in \mathbb{R}^{n}$ is the neuron state vector with $n$ being the number of neurons in NN, $g(y(t))=$ $\left[g_{1}\left(y_{1}(t)\right), \ldots, g_{n}\left(y_{n}(t)\right)\right]^{t} \in \mathbb{R}^{n}$ denotes the neuron activation function, $g(y(t-\tau(t)))=\left[g_{1}\left(y_{1}(t-\tau(t))\right), \ldots, g_{n}\left(y_{n}(t-\right.\right.$ $\tau(t)))]^{t} \in \mathbb{R}^{n}, A_{o}=\operatorname{diag}\left\{a_{o j}\right\} \in \mathbb{R}^{n \times n}$ is a positive diagonal matrix, $W_{o}=\left(W_{j k}^{o}\right) \in \mathbb{R}^{n \times n}$ and $W_{1}=\left(W_{j k}^{1}\right) \in \mathbb{R}^{n \times n}$ are the interconnection matrices representing the weight coefficients of the neurons, $b=\left[b_{1}, \ldots, b_{n}\right]^{t} \in \mathbb{R}^{n}$ is a constant input vector, and $\Delta A(t), \Delta W_{o}(t)$, and $\Delta W_{1}(t)$ are uncertain system matrices of the form

$$
\begin{aligned}
\Delta A_{o} & =E G(t) F, \\
\Delta W_{o} & =E_{o} G_{o}(t) F_{o}, \\
\Delta W_{1} & =E_{1} G_{1}(t) F_{1}, \\
G^{t}(t) G(t) & \leq I, \\
G_{o}^{t}(t) G_{o}(t) & \leq I, \\
G_{1}^{t}(t) G_{1}(t) & \leq I .
\end{aligned}
$$

In the sequel, it is assumed that the delay $\tau(t)$ is a differentiable time-varying function satisfying

$$
\begin{aligned}
0 & <\tau(t) \leq \varrho, \\
\dot{\tau}(t) & \leq \mu,
\end{aligned}
$$

where the bounds $\varrho$ and $\mu$ are known constant scalars. Observe that there is no restriction on the derivative of the 
time-varying delay function $\mu$, thereby allowing fast timedelays to occur. This is in contrast with other methods which places $\mu<1$, thereby limiting the method to slow variations in time-delay.

Assumption 49. The neuron activation functions, $g_{o j}\left(y_{j}(t)\right)$, $j=1, \ldots, n$, and $g_{o j}\left(\left(y_{j}(t-\tau(t))\right)\right), j=1, \ldots, n$, are assumed to be nondecreasing, bounded, globally Lipschitz and satisfy

$$
\begin{aligned}
0 \leq \frac{g_{j}\left(\zeta_{j}\right)-g_{k}\left(\zeta_{k}\right)}{\zeta_{j}-\zeta_{k}} \leq k_{j}, & \\
& \zeta_{j}, \zeta_{k} \in \mathbb{R}, \zeta_{j} \neq \zeta_{k}, j=1, \ldots, n,
\end{aligned}
$$

where $k_{j}, j=1, \ldots, n$, are positive constants.

We note that the existence of an equilibrium point of system (136) is guaranteed by the fixed point theorem. Now let $y^{*}=\left[y_{1}^{*}, \ldots, y_{n}^{*}\right]^{t}$ be an equilibrium of (136), and let

$$
x(t)=y(t)-y^{*} .
$$

It is easy to see that (136) is transformed to

$$
\begin{aligned}
\dot{x}(t)= & -\left[A_{o}+\Delta A_{o}(t)\right] x(t) \\
& +\left[W_{o}+\Delta W_{o}(t)\right] f(x(t)) \\
& +\left[W_{1}+\Delta W_{1}(t)\right] f(x(t-\tau(t))) \\
= & -A_{o \Delta} x(t)+W_{o \Delta} f(x(t)) \\
& +W_{1 \Delta} f(x(t-\tau(t))),
\end{aligned}
$$

where $f(x(t))=\left[f_{1}(x(t)), \ldots, f_{n}(x(t))\right]^{t}$ and $f_{j}\left(x_{j}(t)\right)=$ $g_{j}\left(x_{j}(t)+y_{j}^{*}\right)-g_{j}\left(y_{j}^{*}\right)$ with $f_{j}(0)=0, j=1, \ldots, n$. It is observed that $f(x(t))$ satisfies $f(0, t)=0, \forall t$, and the following condition for all $(x, t), \in \mathbb{R}^{n} \times \mathbb{R}$ :

$$
\begin{gathered}
f(x(t), t) \leq k x, \\
f(x(t-\tau), t) \leq k x(t-\tau),
\end{gathered}
$$

where $k>0$ is a constant. In the absence of uncertainties, we get from (141) the nominal NN model

$$
\dot{x}(t)=-A_{o} x(t)+W_{o} f(x(t))+W_{1} f(x(t-\tau(t))) .
$$

In the sequel, the global delay-dependent asymptotic stability the equilibrium of system (136) is investigated, which corresponds to the uniqueness of the equilibrium point.

The following theorem establishes the main result for global delay-dependent asymptotic stability of the NN system.

Theorem 50. Given $\varrho>0$ and $\mu>0$. System (143) is globally delay-dependent asymptotically stable if there exist weighting matrices $\mathscr{P}>0, \mathbb{Q}>0, \mathscr{R}>0$, and $\mathscr{Z}>0$ and free-weighting parameter matrices $N_{a}, N_{c}, M_{a}, M_{c}, \mathcal{S}>0, \mathscr{M}>0$ satisfying the following LMI:

$$
\Xi=\left[\begin{array}{cccc}
\Xi_{o} & \widehat{\mathscr{P}}_{o} & \widehat{\mathscr{P}}_{c} & \varrho \mathcal{N} \\
\bullet & -2 k^{-1} \mathcal{S} & 0 & 0 \\
\bullet & \bullet & -2 k^{-1} \mathscr{M} & 0 \\
\bullet & \bullet & \bullet & -\varrho \mathscr{Z}
\end{array}\right]<0,
$$

where

$$
\Xi_{o}=\left[\begin{array}{cccc}
\Xi_{o 1} & \Xi_{o 2} & N_{a} & \mathscr{P}-M_{a}^{t}-A_{o}^{t} M_{c} \\
\bullet & \Xi_{o 3} & N_{c} & 0 \\
\bullet & \bullet & -\mathscr{R} & 0 \\
\bullet & \bullet & \bullet & -M_{c}-M_{c}^{t}+\varrho \mathscr{Z}
\end{array}\right],
$$$$
\Xi_{o 1}=-M_{a}^{t} A_{o}-A_{o}^{t} M_{a}^{+} Q+\mathscr{R}+N_{a}+N_{a}^{t} \text {, }
$$$$
\Xi_{o 2}=-2 N_{a}+N_{c}^{t}
$$$$
\Xi_{o 3}=-(1-\mu) Q-2 N_{c}-2 N_{c}^{t} \text {, }
$$$$
\mathcal{N}=\left[\begin{array}{c}
N_{a} \\
N_{c} \\
0 \\
0
\end{array}\right]
$$

$\widehat{\mathscr{P}}_{o}=\left[\begin{array}{c}M_{a}^{t} W_{o}+\mathcal{S} \\ 0 \\ 0 \\ M_{c}^{t} W_{o}\end{array}\right]$,

$$
\widehat{\mathscr{P}}_{c}=\left[\begin{array}{c}
M_{a}^{t} W_{1} \\
\mathscr{M} \\
0 \\
M_{c}^{t} W_{1}
\end{array}\right] \text {. }
$$

On considering the UNN system in (141) with the uncertainty in (137), it follows from Theorem 50 that the UNN system is globally delay-dependent asymptotically stable if there exist weighting matrices $\mathscr{P}>0, \mathbb{Q}>0, \mathscr{R}>$ 0 , and $\mathscr{Z}>0$ and free-weighting parameter matrices $N_{a}, N_{c}, M_{a}, M_{c}, \mathcal{S}>0, \mathscr{M}>0$ satisfying the following LMI:

$$
\Xi_{\Delta}=\left[\begin{array}{cccc}
\Xi_{o \Delta} & \mathscr{P}_{o} & \mathscr{P}_{c} & \varrho \mathcal{N} \\
\bullet & -2 k^{-1} \mathcal{S} & 0 & 0 \\
\bullet & \bullet & -2 k^{-1} \mathscr{M} & 0 \\
\bullet & \bullet & \bullet & -\varrho \mathscr{Z}
\end{array}\right]<0 \text {, }
$$


where

$$
\begin{gathered}
\Xi_{o \Delta}=\left[\begin{array}{cccc}
\Xi_{o 1 \Delta} & \Xi_{o 2} & N_{a} & \mathscr{P}-M_{a}^{t}-A_{o \Delta}^{t} M_{c} \\
\bullet & \Xi_{o 3} & N_{c} & 0 \\
\bullet & \bullet & -\mathscr{R} & 0 \\
\bullet & \bullet & \bullet & -M_{c}-M_{c}^{t}+\varrho \mathscr{Z}
\end{array}\right], \\
\Xi_{o 1 \Delta}=-M_{a}^{t} A_{o \Delta}-A_{o \Delta}^{t} M_{a}+\mathscr{Q}+\mathscr{R}+N_{a}+N_{a}^{t},
\end{gathered}
$$

where $\Xi_{o 2}, \Xi_{o 3}, \mathcal{N}, \mathscr{P}_{o}, \mathscr{P}_{c}$ are given in (145). Applying Fact 1 for some scalars $\varepsilon_{1}>0, \varepsilon_{2}>0$, and $\varepsilon_{3}>0$ and invoking Schur complements, it is easy to show that the following theorem holds.

Theorem 51. System (141) with norm-bounded uncertainty (137) is globally delay-dependent asymptotically stable if there exist weighting matrices $\mathscr{P}>0, \mathbb{Q}>0, \mathscr{R}>0$, and $\mathscr{Z}>$ 0 , free-weighting parameter matrices $N_{a}, N_{c}, M_{a}, M_{c}, \mathcal{S}>$ $0, \mathscr{M}>0$, and scalars $\sigma>0, \kappa>0, \varepsilon_{i}>0, i=1,2,3$, satisfying the following LMI:

$$
\widehat{\Xi}=\left[\begin{array}{cc}
\Xi_{s} & \Xi_{x} \\
\bullet & \Xi_{y}
\end{array}\right]<0,
$$

where

$$
\begin{aligned}
& \Xi_{s}=\left[\begin{array}{cccc}
\Xi_{o s} & \widehat{\mathscr{P}}_{o} & \widehat{\mathscr{P}}_{c} & \varrho N_{a} \\
\bullet & \Xi_{f 1} & 0 & \varrho N_{c} \\
\bullet & \bullet & \Xi_{f 2} & 0 \\
\bullet & \bullet & \bullet & -\varrho \mathscr{W}
\end{array}\right], \\
& \Xi_{o s}=\left[\begin{array}{cccc}
\Xi_{o 1 s} & \Xi_{o 2} & N_{a} & \mathscr{P}-M_{a}^{t}-A^{t} M_{c} \\
\bullet & \Xi_{o 3} & N_{c} & 0 \\
\bullet & \bullet & -\mathscr{R} & 0 \\
\bullet & \bullet & \bullet & -M_{c}-M_{c}^{t}+\varrho \mathscr{X}
\end{array}\right] \text {, } \\
& \Xi_{o 1 s}=-M_{a}^{t} A_{o}-A_{o}^{t} M_{a}+\mathscr{Q}+\mathscr{R}+N_{a}+N_{a}^{t} \\
& +\varepsilon_{1} F^{t} F \text {, } \\
& \Xi_{f 1}=-2 k^{-1} \mathcal{S}+\varepsilon_{2} F_{o}^{t} F_{o}, \\
& \Xi_{f 2}=-2 k^{-1} \mathscr{M}+\varepsilon_{3} F_{1}^{t} F_{1}, \\
& \Xi_{x}=\left[\begin{array}{ccc}
-M_{a}^{t} E & M_{a}^{t} E_{o} & M_{a}^{t} E_{1} \\
0 & 0 & 0 \\
0 & 0 & 0 \\
-M_{c}^{t} E & M_{c}^{t} E_{o} & M_{c}^{t} E_{1} \\
0 & 0 & 0 \\
0 & 0 & 0 \\
0 & 0 & 0
\end{array}\right],
\end{aligned}
$$

$$
\Xi_{y}=\left[\begin{array}{ccc}
-\varepsilon_{1} I & 0 & 0 \\
\bullet & -\varepsilon_{2} I & 0 \\
\bullet & \bullet & -\varepsilon_{3} I
\end{array}\right] .
$$

The reader is referred to [58] for further results on using expanded LKFs.

\section{Networked Control Systems}

Typically in process industries, a network used at the lowest level of a process/factory communication hierarchy is called a fieldbus. Fieldbuses are intended to replace the traditional wiring between sensors, actuators, and controllers. In distributed control system applications, a feedback control loop is often closed through the network, which is called a network-based control system (NBCS); see details in [44, 59-69]. In the NBCS, various delays with variable lengths occurred due to sharing a common network medium, which are called network-induced delays. These delays are dependent on configurations of the network and the given system. Those make the NBCS unstable.

In feedback control systems, it is significant that sampled data must be transmitted within a sampling period and stability of control systems should be guaranteed. While a shorter sampling period is preferable in most control systems, for some cases, it can be lengthened up to a certain bound within which stability of the system is guaranteed in spite of the performance degradation. This certain bound is called a maximum allowable delay bound (MADB). The MADB depends only on parameters and configurations of the given plant and the controller.

In addition, a faster sampling is said to be desirable in sampled-data systems because the performance of the discrete-time system controller can approximate that of the continuous-time system. But in NBCS (see Figure 1), the high sampling rate can increase network load, which in turn results in longer delay of the signals. Thus finding a sampling rate that can both tolerate the network-induced delay and achieve desired system performance is of fundamental importance in the NBCS design.

11.1. State Feedback Stabilization. Consider the plant model described as

$$
\begin{aligned}
\dot{x}(t) & =A x(t)+B u(t), \\
t & \\
y(t) & =C x(t), \\
u\left(t^{+}\right) & =-K_{i} x\left(t-\tau_{k}\right), \\
& t \in\left\{k h+\tau_{k},(k+1) h+\tau_{k+1}\right), \\
&
\end{aligned}
$$

Sampling the above system with period $h$ and defining $z(k h)=\left[x^{T}(h), u^{T}((k-1) h)\right]^{T}$ yielded the following closedloop system:

$$
z((k+1) h)=\widetilde{\Phi}\left(K_{i}\right) z(k h) \quad \forall i=1,2, \ldots, p .
$$




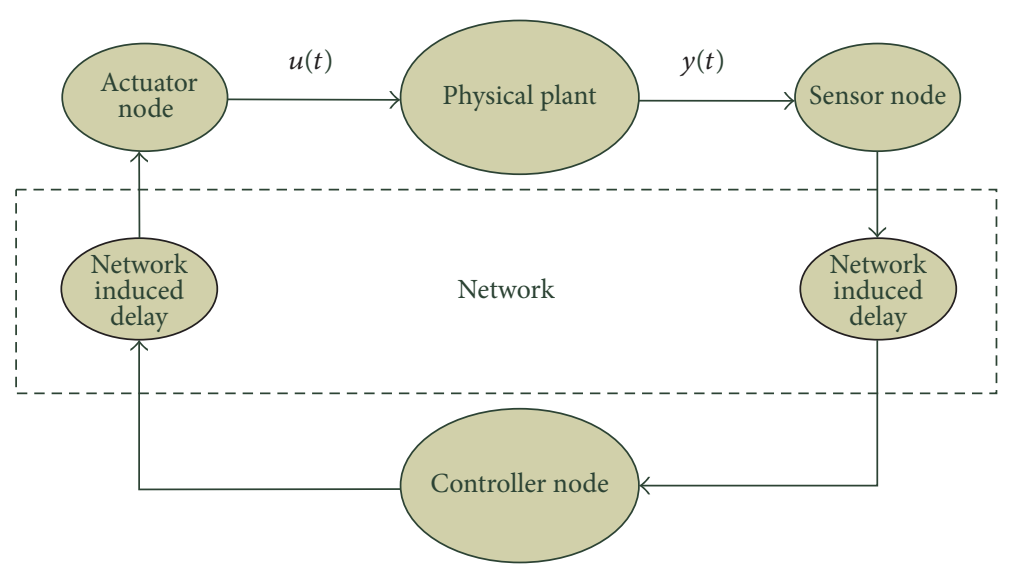

FIGURE 1: A feedback control loop with network-induced delays.

A recent survey of the stabilization methods is reported in [44].

11.2. Observer-Based Feedback Stabilization. An observerbased stabilizing controller can be designed for networked systems involving both random measurement and actuation delays. The LTI plant under consideration was assumed to be of the form

$$
\begin{gathered}
x_{p}(k+1)=A x_{p}+B u_{p}, \\
y_{p}=C x_{p},
\end{gathered}
$$

where $x_{p}(k) \in \mathfrak{R}^{n}$ is the state vector and $u_{p}(k) \in \mathfrak{R}^{m}$ and $y_{p}(k) \in \Re^{p}$ are the control input and output vectors of the plant, respectively. The measurement subjected to random communication delay is given by

$$
y_{c}(k)=(1-\delta(k)) y_{p}(k)+\delta(k) y_{p}\left(k-\tau_{k}^{m}\right),
$$

where $\tau_{k}^{m}$ is the measurement delay, whose occurrence is governed by the Bernoulli distribution, and $\delta(k)$ is Bernoulli distributed sequence with

$$
\begin{aligned}
& \operatorname{Prob}\{\delta(k)=1\}=\mathbb{E}\{\delta(k)\}=\bar{\delta}, \\
& \operatorname{Prob}\{\delta(k)=0\}=1-\mathbb{E}\{\delta(k)\}=1-\bar{\delta} .
\end{aligned}
$$

The following observer-based controller is designed when the full state vector is not available.

\section{Observer}

$$
\begin{gathered}
\widehat{x}(k+1)=A \widehat{x}+B u_{c}(k)+L\left(y_{c}(k)-\widehat{y}_{c}(k)\right), \\
\widehat{y}_{c}(k)=(1-\bar{\delta}) C \widehat{x}(k)+\bar{\delta} C \widehat{x}\left(k-\tau_{k}^{m}\right) .
\end{gathered}
$$

\section{Controller}

$$
\begin{aligned}
u_{c}(k) & =K \hat{x}(k) \\
u_{p} & =(1-\alpha) u_{c}(k)+\alpha u_{c}\left(k-\tau_{k}^{a}\right),
\end{aligned}
$$

where $\widehat{x}(k) \in \Re^{n}$ is the estimate of system (152), $\widehat{y}_{c}(k) \in \mathfrak{R}^{p}$ is the observer output, and $L \in \mathfrak{R}^{n \times p}$ and $K \in \mathfrak{R}^{m \times n}$ are the observer gain and the controller gain, respectively. The stochastic variable $\alpha$, mutually independent of $\delta$, is also a Bernoulli distributed white sequence with

$$
\begin{aligned}
& \operatorname{Prob}\{\alpha(k)=1\}=\mathbb{E}\{\alpha(k)\}=\bar{\alpha}, \\
& \operatorname{Prob}\{\alpha(k)=0\}=1-\mathbb{E}\{\alpha(k)\}=1-\bar{\alpha},
\end{aligned}
$$

where $\tau_{k}^{a}$ is the actuation delay. It is assumed that $\tau_{k}^{a}$ and $\tau_{k}^{m}$ are time-varying and have the following bounded condition:

$$
\begin{gathered}
\underline{d}_{m} \leq \tau_{k}^{m} \leq \bar{d}_{m}, \\
\underline{d}_{a} \leq \tau_{k}^{a} \leq \bar{d}_{a} .
\end{gathered}
$$

The estimation error is defined by

$$
e(k)=x_{p}(k)-\widehat{x}(k) .
$$

This yields

$$
\begin{aligned}
x_{p}(k+1)=[ & A+(1-\bar{\alpha}) B K] x_{p}(k) \\
& -(1-\bar{\alpha}) B K e(k)+\bar{\alpha} B K x_{p}\left(k-\tau_{k}^{a}\right) \\
& -\bar{\alpha} B K e\left(k-\tau_{k}^{a}\right) \\
& -(\alpha-\bar{\alpha}) B K x_{p}(k) \\
& +(\alpha-\bar{\alpha}) B K e(k) \\
& +(\alpha-\bar{\alpha}) B K x_{p}\left(k-\tau_{k}^{a}\right) \\
& -(\alpha-\bar{\alpha}) B K e\left(k-\tau_{k}^{a}\right), \\
e(k+1)=[ & A-(1-\bar{\delta}) L C] e(k) \\
& -\bar{\delta} L C e\left(k-\tau_{k}^{m}\right) \\
& +(\delta-\bar{\delta}) L C x_{p}(k) \\
& -(\delta-\bar{\delta}) L C x_{p}\left(k-\tau_{k}^{m}\right) .
\end{aligned}
$$




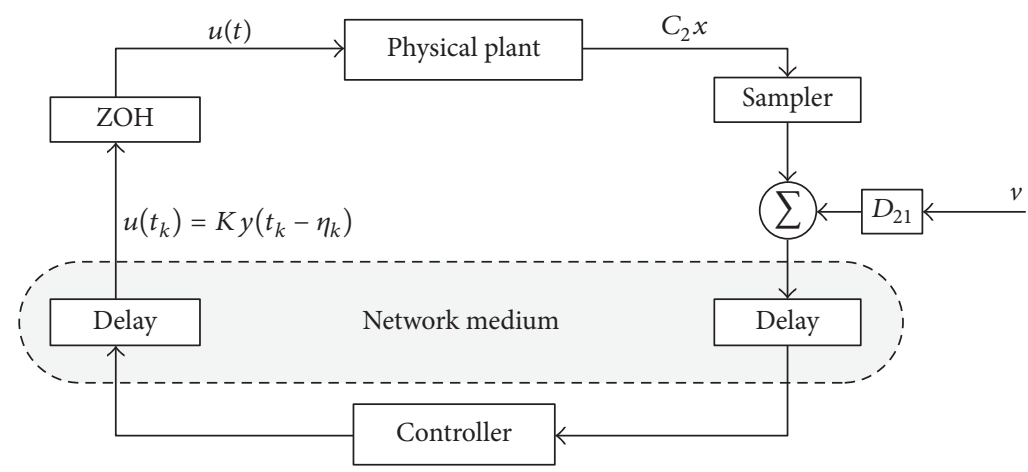

FIGURE 2: Networked output-feedback control system.

System (160) is equivalent to the following compact form:

$$
\begin{aligned}
\varepsilon(k+1)= & (\bar{A}+\widetilde{A}) \varepsilon(k)+(\bar{B}+\widetilde{B}) \varepsilon\left(k-\tau_{k}^{m}\right) \\
& +(\bar{C}-\widetilde{C}) \varepsilon\left(k-\tau_{k}^{a}\right),
\end{aligned}
$$

where

$$
\begin{aligned}
& \varepsilon(k)=\left[\begin{array}{ll}
x_{p}^{T}(k) & e^{T}(k)
\end{array}\right]^{T}, \\
& \bar{A}=\left[\begin{array}{cc}
A+(1-\bar{\alpha}) B K & -(1-\bar{\alpha}) B K \\
0 & A-(1-\bar{\delta}) L C
\end{array}\right], \\
& \widetilde{A}=\left[\begin{array}{cc}
-(\alpha-\bar{\alpha}) B K & (\alpha-\bar{\alpha}) B K \\
(\delta-\bar{\delta}) L C & 0
\end{array}\right] \text {, } \\
& \bar{B}=\left[\begin{array}{cc}
0 & 0 \\
0 & -\bar{\delta} L C
\end{array}\right] \text {, } \\
& \widetilde{B}=\left[\begin{array}{cc}
0 & 0 \\
-(\delta-\bar{\delta}) L C & 0
\end{array}\right] \text {, } \\
& \bar{C}=\left[\begin{array}{cc}
\bar{\alpha} B K & -\bar{\alpha} B K \\
0 & 0
\end{array}\right] \text {, } \\
& \widetilde{C}=\left[\begin{array}{cc}
(\alpha-\bar{\alpha}) B K & -(\alpha-\bar{\alpha}) B K \\
0 & 0
\end{array}\right] \text {. }
\end{aligned}
$$

Remark 52. It is noted that a majority of the existing works on the stability of NCS (in the framework of time-delay approach) are reduced to some Lyapunov-based analysis of systems with uncertain and bounded time-varying delays; see [44]. In the following sections, we will present alternative approaches that will lead to improved results.

11.3. Lyapunov-Based Sampled-Data Stabilization. Three main approaches have been used to the sampled-data control and later to the Networked Control Systems (NCS), where the plant is controlled via communication network:

(A) The first one is based on discrete-time models [70, 71]. This approach is not applicable to the performance analysis (like the exponential decay rate) of the resulting continuous-time closed-loop system.

(B) The second one is a time-delay approach, where the system is modeled as a continuous-time system with a time-varying sawtooth delay in the control input $[8,72-74]$. The time-delay approach via time-independent Lyapunov-Krasovskii functionals or Lyapunov-Razumikhin functions leads to linear matrix inequalities (LMIs) for analysis and design of linear uncertain NCS.

(C) The third approach is based on the representation of the sampled-data system in the form of impulsive model $[72,73]$. Recently, the impulsive model approach was extended to the case of uncertain sampling intervals [75] by employing a discontinuous Lyapunov function method, which improved the existing Lyapunov-based results. Recently, the latter result was recovered via an input-output approach by application of the vector extension of Wirtinger's inequality [76].

Consider the continuous-time system depicted in Figure 2:

$$
\begin{aligned}
& \dot{x}(t)=A x(t)+B u(t)+E w(t), \\
& z(t)=C_{1} x(t)+D_{1} u(t),
\end{aligned}
$$

where $x(t) \in \mathbb{R}^{n}$ is the state, $z(t) \in \mathbb{R}^{r}$ is the signal to be controlled or estimated, $w(t) \in \mathbb{R}^{q}$ is the disturbance, $u(t) \in$ $\mathbb{R}^{m}$ is the control input, and $A(t) \in \mathbb{R}^{n \times n}, B(t) \in \mathbb{R}^{n \times m}, C_{1} \in$ $\mathbb{R}^{p \times n}, D_{1} \in \mathbb{R}^{p \times m}$, and $E \in \mathbb{R}^{n \times q}$ are system matrices.

In Figure 2, the sampler is time-driven, whereas the controller and the Zero-Order Hold $(\mathrm{ZOH})$ are event-driven (in the sense that the controller and the $\mathrm{ZOH}$ update their outputs as soon as they receive a new sample). For simplicity in exposition, we assume that the measurement output $y\left(s_{k}\right) \in \mathbb{R}^{p}$ is available at discrete sampling instants

$$
0=s_{0}<s_{1}<\cdots<s_{k}<\cdots, \quad \lim _{k \rightarrow \infty} s_{k}=\infty,
$$

and it may be corrupted by a measurement noise signal $v\left(s_{k}\right)$ :

$$
y(t)=C x(t)+D u(t) .
$$




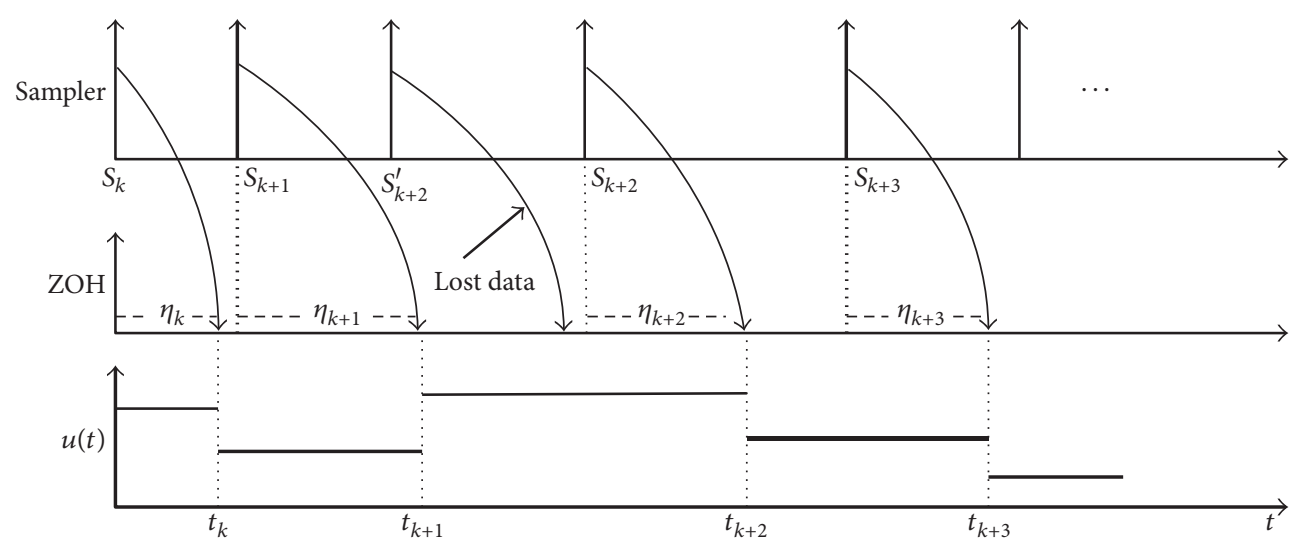

FIGURE 3: NCS timing diagram.

By considering nonuniform sampling, data packet dropouts can be accommodated. In this respect, $y\left(s_{k}\right), k=$ $0,1,2, \ldots$, correspond to the measurements that are not lost. The timing diagram of the considered NCS with both delay and packet dropout is shown in Figure 3, where $s_{k}=t_{k}-\lambda_{k}$ accounts for the sampling time of the data that has not been lost. In this setup, $t_{k}$ denotes the updating instant time of the $\mathrm{ZOH}$, and suppose that the updating signal at the instant $t_{k}$ has experienced a signal transmission delay $\lambda_{k}$. Adopting the approach of [75], we allow the delays $\lambda_{k}$ to grow larger than $s_{k+1}-s_{k}$, provided that the sequence of input update times $t_{k}$ remains strictly increasing. This implies that if an old sample gets to the destination after the most recent one, it should be dropped.

The static output-feedback controller has a form

$$
u\left(t_{k}\right)=K_{o} y\left(t_{k}-\lambda_{k}\right), \quad t_{k} \leq t \leq t_{k+1},
$$

where $K_{o}$ is the controller gain and $t_{k+1}$ is the next updating instant time of the $\mathrm{ZOH}$ after $t_{k}$. It is known that

$$
t_{k+1}-t_{k}+\lambda_{k} \leq \tau_{M}, \quad 0 \leq \lambda_{k} \leq \lambda_{M}, k=0,1,2, \ldots,
$$

where $\lambda_{M}$ is a known upper bound on the network-induced delays $\lambda_{k}$ and $\tau_{M}$ denotes the maximum time span between the time $s_{k}=t_{k}-\lambda_{k}$ at which the state is sampled, and the time $t_{k+1}$ at which next update arrives at the ZOH. Observe that the sampling intervals and the numbers of successive packet dropouts are uniformly bounded.

Within the foregoing representation, exponential stability, state feedback, and static output-feedback results are developed in [77]. More elaborate results can be found in [74].

\section{Interconnected Systems}

We consider a class of linear systems $S$ structurally composed of $n_{s}$ coupled subsystems $S_{j}$ depicted in Figure 4 and modeled by the state-space model:

$$
\begin{aligned}
\dot{x}_{j}(t)= & A_{j \Delta} x_{j}(t)+A_{d j \Delta} x_{j}\left(t-\tau_{j}\right)+B_{j \Delta} u_{j}(t) \\
& +c_{j}(k)+\Gamma_{j \Delta} w_{j}(t),
\end{aligned}
$$

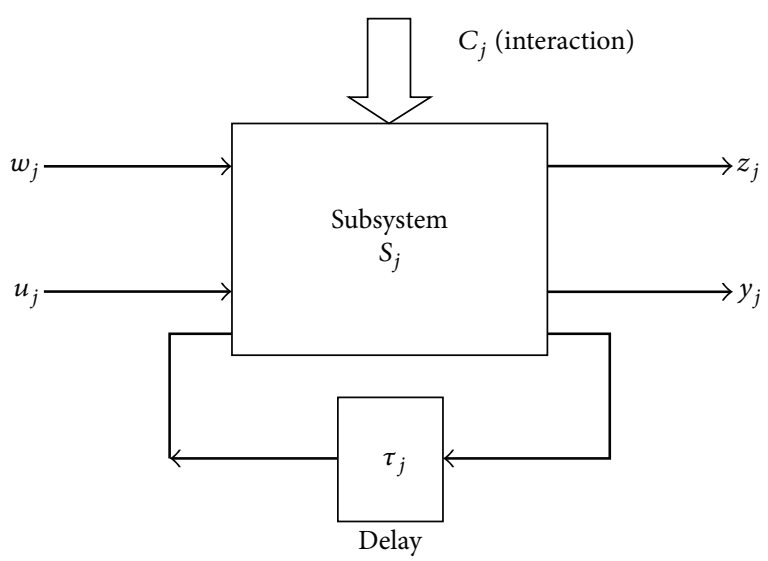

Figure 4: Subsystem model.

$$
\begin{aligned}
z_{j}(t)= & G_{j \Delta} x_{j}(t)+G_{d j \Delta} x\left(t-\tau_{j}\right)+D_{j \Delta} u_{j}(t) \\
& +\Phi_{j \Delta} w_{j}(t), \\
y_{j}(t)= & C_{j \Delta} x_{j}(t)+C_{d j \Delta} x\left(t-\tau_{j}\right), \\
c_{j}(t)= & \sum_{k=1}^{n_{s}} F_{j k \Delta} x_{k}(t)+\sum_{k=1}^{n_{s}} E_{j k \Delta} x_{k}\left(t-\eta_{j k}(t)\right)
\end{aligned}
$$

whose matrices are containing uncertainties which belong to a real convex bounded polytopic model of the type

$$
\begin{aligned}
& {\left[\begin{array}{llll}
A_{j \Delta} & A_{d j \Delta} & B_{j \Delta} & \Gamma_{j \Delta} \\
G_{j \Delta} & G_{d j \Delta} & D_{j \Delta} & \Phi_{j \Delta} \\
C_{j \Delta} & C_{d j \Delta} & E_{j k \Delta} & F_{j k \Delta}
\end{array}\right] \in \Pi_{\lambda}} \\
& \quad \triangleq\left\{\left[\begin{array}{llll}
A_{j \lambda} & A_{d j \lambda} & B_{j \lambda} & \Gamma_{j \lambda} \\
G_{j \lambda} & G_{d j \lambda} & D_{j \lambda} & \Phi_{j \lambda} \\
C_{j \lambda} & C_{d j \lambda} & E_{j k \lambda} & F_{j k \lambda}
\end{array}\right]\right. \\
& \left.=\sum_{s=1}^{N} \lambda_{s}\left[\begin{array}{llll}
A_{j s} & A_{d j s} & B_{j s} & \Gamma_{j s} \\
G_{j s} & G_{d j s} & D_{j s} & \Phi_{j s} \\
C_{j s} & C_{d j s} & E_{j k s} & F_{j k s}
\end{array}\right], \lambda_{s} \in \Lambda\right\},
\end{aligned}
$$


where $\Lambda$ is the unit simplex:

$$
\Lambda \triangleq\left\{\left(\lambda_{1}, \ldots, \lambda_{N}\right): \sum_{j=1}^{N} \lambda_{j}=1, \lambda_{j} \geq 0\right\}
$$

Define the vertex set $\mathcal{N}=\{1, \ldots, N\}$. We use $\{A, \ldots, \Phi\}$ to imply generic system matrices and $\left\{A_{j}, \ldots, \Phi_{j}, j \in \mathcal{N}\right\}$ to represent the respective values at the vertices.

In the absence of uncertainties, system (168) reduces to the nominal state-space model

$$
\begin{aligned}
\dot{x}_{j}(t)= & A_{j} x_{j}(t)+A_{d j} x_{j}\left(t-\tau_{j}(t)\right)+B_{j} u_{j}(t) \\
& +c_{j}(t)+\Gamma_{j} w_{j}(t), \\
z_{j}(t)= & G_{j} x_{j}(t)+G_{d j} x_{j}\left(t-\tau_{j}(t)\right)+D_{j} u_{j}(t) \\
& +\Phi_{j} w_{j}(t), \\
y_{j}(t)= & C_{j} x_{j}(t)+C_{d j} x_{j}\left(t-\tau_{j}(t)\right), \\
c_{j}(t)= & \sum_{k=1}^{n_{s}} F_{j k} x_{k}(t)+\sum_{k=1}^{n_{s}} E_{j k} x_{k}\left(t-\eta_{j k}(t)\right),
\end{aligned}
$$

where, for $j \in\left\{1, \ldots, n_{s}\right\}, \quad x_{j}(t) \in \mathfrak{R}^{n_{j}}$ is the state vector, $u_{j}(t) \in \mathfrak{R}^{m_{j}}$ is the control input, $y_{j}(t) \in \mathfrak{R}^{p_{j}}$ is the measured output, $w_{j}(t) \in \Re^{q_{j}}$ is the disturbance input which belongs to $\mathscr{L}_{2}[0, \infty), z_{j}(t) \in \mathfrak{R}^{q_{j}}$ is the performance output, $c_{j}(t) \in$ $\mathfrak{R}^{n_{j}}$ is the coupling vector, and $\tau_{j}, \eta_{j k}, j, k \in\left\{1, \ldots, n_{s}\right\}$, are unknown time-delay factors satisfying

$$
\begin{aligned}
& 0 \leq \tau_{j}(t) \leq \varrho_{j}, \quad \dot{\tau}_{j}(t) \leq \mu_{j}, \\
& 0 \leq \eta_{j k}(t) \leq \varrho_{j k}, \quad \dot{\eta}_{j k}(t) \leq \mu_{j k},
\end{aligned}
$$

where the bounds $\varrho_{j}, \varrho_{j k}, \mu_{j}, \mu_{j k}$ are known constants in order to guarantee smooth growth of the state trajectories. The matrices $A_{j} \in \mathfrak{R}^{n_{j} \times n_{j}}, B_{j} \in \mathfrak{R}^{n_{j} \times m_{j}}, D_{j} \in \mathfrak{R}^{q_{j} \times m_{j}}, A_{d j} \in$ $\mathfrak{R}^{n_{j} \times n_{j}}, \Phi_{j} \in \mathfrak{R}^{q_{j} \times q_{j}}, \Gamma_{j} \in \mathfrak{R}^{n_{j} \times q_{j}}, C_{j} \in \mathfrak{R}^{p_{j} \times n_{j}}, C_{d j} \in \mathfrak{R}^{p_{j} \times n_{j}}$, $G_{j} \in \mathfrak{R}^{q_{j} \times n_{j}}, G_{d j} \in \mathfrak{R}^{q_{j} \times n_{j}}, F_{j k} \in \mathfrak{R}^{n_{j} \times n_{k}}$, and $E_{j k} \in \mathfrak{R}^{n_{j} \times n_{k}}$ are real and constants. The initial condition $\left\langle x_{j}(0), x_{j}(r)\langle=\right.$ $\left\langle x_{o j}, \phi_{j}\left\langle, j \in\left\{1, \ldots, n_{s}\right\}\right.\right.$, where $\phi_{j}(\cdot) \in \mathscr{L}_{2}\left[-\tau_{j}^{*}, 0\right], j \in$ $\left\{1, \ldots, n_{s}\right\}$. The inclusion of the terms $A_{d j} x_{j}\left(t-\tau_{j}(t)\right)$ and $E_{j k} x_{k}\left(t-\eta_{j k}(t)\right)$ is meant to emphasize the delay within each subsystem (local delay) and among the subsystems (coupling delay), respectively.

We develop new criteria for LMI-based characterization of delay-dependent asymptotic stability and $\mathscr{L}_{2}$ gain analysis which requires only subsystem information thereby assuring decentralization. The criteria include some parameter matrices aims at expanding the range of applicability of the developed conditions. The following theorem establishes the main result subsystem $S_{j}$.

Theorem 53. Give $\varrho_{j}>0, \mu_{j}>0, \varrho_{j k}>0$, and $\mu_{j k}>0, j, k=$ $1, \ldots, n_{s}$. The family of nominal subsystems $\left\{\mathrm{S}_{j}\right\}$ with $u_{j}(\cdot) \equiv 0$ where $\mathrm{S}_{\mathrm{j}}$ is described by (171) is delay-dependent asymptotically stable with $\mathscr{L}_{2}$-performance bound $\gamma_{j}, j=1, \ldots, n_{s}$, if there exist positive-definite matrices $\mathscr{P}_{j}, \mathscr{Q}_{j}, \mathscr{W}_{j}, \mathscr{Z}_{k j}, k, j=$ $1, \ldots, n_{s}$, and parameter matrices $\Theta_{j}$ and $\Upsilon_{j}$ satisfying the following LMIs for $j=1, \ldots, n_{s}$

$$
\left[\begin{array}{ccccccc}
\Xi_{o j} & \Xi_{a j} & -\varrho_{j} \Theta_{j} & \Xi_{c j} & \mathscr{P}_{j} \Gamma_{j} & G_{j}^{t} & \varrho_{j} A_{j}^{t} \mathscr{W}_{j} \\
\bullet & -\Xi_{m j} & -\varrho_{j} \Upsilon_{j} & 0 & 0 & G_{d j}^{t} & \varrho_{j} A_{d_{j}}^{t} \mathscr{W}_{j} \\
\bullet & \bullet & -\varrho_{j} \mathscr{W}_{j} & 0 & 0 & 0 & 0 \\
\bullet & \bullet & \bullet & -\Xi_{n j} & 0 & 0 & \varrho_{j} \sum_{k=1}^{n_{s}} E_{k j} \mathscr{W}_{j} \\
\bullet & \bullet & \bullet & \bullet & -\gamma_{j}^{2} I_{j} & \Phi_{j}^{t} & \varrho_{j} \Gamma_{j}^{t} \mathscr{W}_{j} \\
\bullet & \bullet & \bullet & \bullet & \bullet & -I_{j} & 0 \\
\bullet & \bullet & \bullet & \bullet & \bullet & \bullet & -\varrho_{j} \mathscr{W}_{j}
\end{array}\right]
$$

where

$$
\begin{aligned}
\Xi_{o j}= & \mathscr{P}_{j}\left[A_{j}+\sum_{k=1}^{n_{s}} F_{k j}\right]+\left[A_{j}+\sum_{k=1}^{n_{s}} F_{k j}\right]^{t} \mathscr{P}_{j}^{t}+\Theta_{j} \\
& +\Theta_{j}^{t}+\mathscr{Q}_{j}+\sum_{k=1}^{n_{s}} \mathscr{Z}_{k j}, \\
\Xi_{m j}= & \Upsilon_{j}+\Upsilon_{j}^{t}+\left(1-\mu_{j}\right) \mathscr{Q}_{j}, \\
\Xi_{a j}= & \mathscr{P}_{j} A_{d j}-\Theta_{j}+\Upsilon_{j}^{t}, \\
\Xi_{n j}= & \sum_{k=1}^{n_{s}}\left(1-\mu_{k j}\right) \mathscr{Z}_{k j}, \\
\Xi_{c j}= & \mathscr{P}_{j} \sum_{k=1}^{n_{s}} E_{k j} .
\end{aligned}
$$

More detailed results can be found in [78].

\section{Conclusions and Future Work}

This paper has overviewed the research area of stability and stabilization of systems with time-delays with emphasis on the following topics:

(i) Systems with time-delays constitute a good compromise between the too simple models with finite dimension and the great complexity of PDEs. The behavior features and the structural characteristics of delay systems are particular enough to justify specific techniques.

(ii) The main Lyapunov-based tools have to be used developing robust stability in combination with model transformations. Several extensions are anticipated when examining different forms of LyapunovKrasovskii functionals.

(iii) In the robust control area, existing results can be generally subdivided into two classes: 
(C1) The first class consists in systems with input or output delays (mainly, $\mathscr{H}_{\infty}$ performance or predictor-like techniques).

(C2) The second class in state delays (discrete or distributed). The intersection of the two classes is still to be addressed.

(iv) Many contemporary dynamical systems with aftereffect are still requiring further investigation: this is the case, for instance, of delay systems with strong nonlinearities, as well as time-varying or state-dependent delays.

(v) There are classes of nonlinear dynamical systems with delays including jump systems, fuzzy systems, and switched systems inviting additional research efforts.

(vi) Recently, a surge of interests has been recently arisen regarding Wirtinger-based integral inequality and augmented Lyapunov-Krasovskii functionals [7983]. The ensuing results triggered recent development in the time-delay system stability. Further discussions and assessments of these results and related issues suggest attractive research directions at least from the computational standpoint.

(vii) The class of uncertain nonlinear networked systems with both multiple stochastic time-varying communication delays and multiple packet dropouts was addressed in [84] for filtering design and in [85] for reliable control. A promising research direction is to extend the role of delay patterns to alternative forms.

\section{Conflicts of Interest}

The author declares that there are no conflicts of interest regarding the publication of this paper.

\section{Acknowledgments}

This research work is supported by the Deanship of Scientific Research (DSR) at KFUPM through research Project no. IN 141048.

\section{References}

[1] V. L. Kharitonov, "Robust stability analysis of time delay systems: a survey," in Proceedings of the 4th IFAC Conference on System Structure and Control, pp. 1-12, Nantes, France, 1998.

[2] V. L. Kharitonov and D. Melchor-Aguilar, "On delay-dependent stability conditions," Systems \& Control Letters, vol. 40, no. 1, pp. 71-76, 2000.

[3] J. K. Hale and S. M. Verduyn Lunel, Introduction to FunctionalDifferential Equations, vol. 99 of Applied Mathematical Sciences, Springer, New York, NY, USA, 1993.

[4] K. Gu, V. L. Kharitonov, and J. Chen, Stability of Time-Delay Systems, Control Engineering, Brikhäuser, Boston, Mass, USA, 2003.

[5] J.-P. Richard, “Time-delay systems: an overview of some recent advances and open problems," Automatica, vol. 39, no. 10, pp. 1667-1694, 2003.
[6] M. S. Mahmoud, Robust Control and Filtering for Time-Delay Systems, Marcel Dekker, New York, NY, USA, 2000.

[7] A. Halanay, Differential Equations: Stability, Oscillations, Time Lags, Academic Press, New York, NY, USA, 1966.

[8] E. Fridman, Introduction to Time-delay Systems: Analysis and Control, Springer, Berlin, Germany, 2014.

[9] M. S. Mahmoud, Switched Time-Delay Systems, Springer, Boston, Mass, USA, 2010.

[10] E. F. Infante, "A Liapunov functional for a matrix differencedifferential equation," Journal of Differential Equations, vol. 29, no. 3, pp. 439-451, 1978.

[11] W. Z. Huang, "Generalization of Liapunov's theorem in a linear delay system," Journal of Mathematical Analysis and Applications, vol. 142, no. 1, pp. 83-94, 1989.

[12] J. Louisell, "A stability analysis for a class of differentialdelay equations having time-varying delay," in Delay Differential Equations and Dynamical Systems, S. Busenberg and M. Martelli, Eds., vol. 1475 of Lecture Notes in Mathematics, pp. 225-242, Springer, Berlin, Germany, 1991.

[13] M. S. Mahmoud and N. F. Al-Muthairi, "Design of robust controllers for time-delay systems," IEEE Transactions on Automatic Control, vol. 39, no. 5, pp. 995-999, 1994.

[14] M. S. Mahmoud and N. F. Al-Muthairi, "Quadratic stabilization of continuous time systems with state-delay and norm-bounded time-varying uncertainties," IEEE Transactions on Automatic Control, vol. 39, no. 10, pp. 2135-2139, 1994.

[15] K. Gu, "A generalized discretization scheme of Lyapunov functional in the stability problem of linear uncertain timedelay systems," International Journal of Robust and Nonlinear Control, vol. 9, no. 1, pp. 1-14, 1999.

[16] K. Gu, "Discretization schemes for Lyapunov-Krasovskii functionals in time-delay systems," Kybernetika, vol. 37, no. 4, pp. 479-504, 2001

[17] X.-J. Jing, D.-L. Tan, and Y.-C. Wang, "An LMI approach to stability of systems with severe time-delay," IEEE Transactions on Automatic Control, vol. 49, no. 7, pp. 1192-1195, 2004.

[18] S. Xu and T. Chen, "Robust $H_{\infty}$ control for uncertain discretetime systems with time-varying delays via exponential output feedback controllers," Systems \& Control Letters, vol. 51, no. 3-4, pp. 171-183, 2004.

[19] S. K. Nguang, P. Shi, and S. Ding, "Delay-dependent fault estimation for uncertain time-delay nonlinear systems: an LMI approach," International Journal of Robust and Nonlinear Control, vol. 16, no. 18, pp. 913-933, 2006.

[20] G.-P. Liu, Y. Q. Xia, J. Chen, D. Rees, and W. S. Hu, "Networked predictive control of systems with random network delays in both forward and feedback channels," IEEE Transactions on Industrial Electronics, vol. 54, no. 3, pp. 1282-1297, 2007.

[21] W.-A. Zhang and L. Yu, "A robust control approach to stabilization of networked control systems with time-varying delays," Automatica, vol. 45, no. 10, pp. 2440-2445, 2009.

[22] X.-M. Sun, G.-P. Liu, W. Wang, and D. Rees, "Stability analysis for networked control systems based on average dwell time method," International Journal of Robust and Nonlinear Control, vol. 20, no. 15, pp. 1774-1784, 2010.

[23] X.-G. Yu, "An LMI approach to robust $\mathrm{H}_{\infty}$ filtering for uncertain systems with time-varying distributed delays," Journal of the Franklin Institute, vol. 345, no. 8, pp. 877-890, 2008.

[24] C. Peng and Y.-C. Tian, "Delay-dependent robust $H_{\infty}$ control for uncertain systems with time-varying delay," Information Sciences, vol. 179, no. 18, pp. 3187-3197, 2009. 
[25] Y. Xia, M. Fu, H. Yang, and G.-P. Liu, "Robust sliding-mode control for uncertain time-delay systems based on delta operator," IEEE Transactions on Industrial Electronics, vol. 56, no. 9, pp. 3646-3655, 2009.

[26] M. F. Miranda and V. J. S. Leite, "Robust stabilization of polytopic discrete-time systems with time-varying state delay: a convex approach," Journal of the Franklin Institute, vol. 348, no. 4, pp. 568-588, 2011.

[27] F. Yang, Z. Wang, Y. S. Hung, and M. Gani, " $H_{\infty}$ control for networked systems with random communication delays," IEEE Transactions on Automatic Control, vol. 51, no. 3, pp. 511-518, 2006.

[28] W.-A. Zhang and L. Yu, "Modelling and control of networked control systems with both network-induced delay and packetdropout," Automatica, vol. 44, no. 12, pp. 3206-3210, 2008.

[29] H. Song, W.-A. Zhang, and L. Yu, " $\mathrm{H}_{\infty}$ filtering of networkbased systems with communication constraints," IET Signal Processing, vol. 4, no. 1, pp. 69-77, 2010.

[30] D. Wu, J. Wu, S. Chen, and J. Chu, "Stability of networked control systems with polytopic uncertainty and buffer constraint," IEEE Transactions on Automatic Control, vol. 55, no. 5, pp. 12021208, 2010.

[31] S. Boyd, L. El Ghaoui, E. Feron, and V. Balakrishnan, Linear Matrix Inequalities in System and Control Theory, vol. 15 of SIAM Studies in Applied Mathematics, SIAM, Philadelphia, Pa, USA, 1994.

[32] E. Fridman and U. Shaked, "An improved stabilization method for linear time-delay systems," IEEE Transactions on Automatic Control, vol. 47, no. 11, pp. 1931-1937, 2002.

[33] E. Fridman and U. Shaked, "Delay-dependent stability and $H_{\infty}$ control: constant and time-varying delays," International Journal of Control, vol. 76, no. 1, pp. 48-60, 2003.

[34] M. Wu, Y. He, J.-H. She, and G.-P. Liu, "Delay-dependent criteria for robust stability of time-varying delay systems," Automatica, vol. 40, no. 8, pp. 1435-1439, 2004.

[35] Y. He, Q.-G. Wang, C. Lin, and M. Wu, "Augmented Lyapunov functional and delay-dependent stability criteria for neutral systems," International Journal of Robust and Nonlinear Control, vol. 15, no. 18, pp. 923-933, 2005.

[36] Y. He, Q.-G. Wang, L. Xie, and C. Lin, "Further improvement of free-weighting matrices technique for systems with timevarying delay," IEEE Transactions on Automatic Control, vol. 52, no. 2, pp. 293-299, 2007.

[37] J. Sun, G. P. Liu, and J. Chen, "Delay-dependent stability and stabilization of neutral time-delay systems," International Journal of Robust and Nonlinear Control, vol. 19, no. 12, pp. 13641375, 2009.

[38] J. Sun and G. P. Liu, "On improved delay-dependent stability criteria for neutral time-delay systems," European Journal of Control, vol. 15, no. 6, pp. 613-623, 2009.

[39] P. Park, "A delay-dependent stability criterion for systems with uncertain time-invariant delays," IEEE Transactions on Automatic Control, vol. 44, no. 4, pp. 876-877, 1999.

[40] Y. S. Moon, P. Park, W. H. Kwon, and Y. S. Lee, "Delaydependent robust stabilization of uncertain state-delayed systems," International Journal of Control, vol. 74, no. 14, pp. 14471455, 2001.

[41] V. Suplin, E. Fridman, and U. Shaked, " $H_{\infty}$ control of linear uncertain time-delay systems-a projection approach," IEEE Transactions on Automatic Control, vol. 51, pp. 680-685, 2006.
[42] K. Gu, "An integral inequality in the stability problem of timedelay systems," in Proceedings of the 39th IEEE Conference on Decision and Control, pp. 2805-2810, Sydney, Australia, 2000.

[43] M. S. Mahmoud and A. Y. Al-Rayyah, "Efficient parameterisation to stability and feedback synthesis of linear time-delay systems," IET Control Theory \& Applications, vol. 3, no. 8, pp. 1107-1118, 2009.

[44] M. S. Mahmoud, Control and Estimation Methods over Communication Networks, Springer, London, UK, 2014.

[45] F. Gouaisbaut and D. Peaucelle, "Delay-dependent robust stability of time delay systems," in Proceedings of the 5th IFAC Symposium on Robust Control Design (ROCOND '06), pp. 453458, Toulouse, France, July 2006.

[46] W.-H. Chen, Z.-H. Guan, and X. Lu, "Delay-dependent guaranteed cost control for uncertain discrete-time systems with delay," IEE Proceedings: Control Theory and Applications, vol. 150, no. 4, pp. 412-416, 2003.

[47] E.-K. Boukas, "Discrete-time systems with time-varying time delay: stability and stabilizability," Mathematical Problems in Engineering, vol. 2006, Article ID 42489, 10 pages, 2006.

[48] V. J. S. Leite, S. Tarbouriech, and P. L. D. Peres, "A convex approach for robust state-feedback control of discrete-time systems with state delay," in Proceedings of the American Control Conference (AAC '04), pp. 2870-2875, Boston, Mass, USA, June 2004.

[49] M. S. Mahmoud, "New results on robust control design of discrete-time uncertain systems," IEE Proceedings-Control Theory and Applications, vol. 152, pp. 453-459, 2005.

[50] Z. Wang, G. Wei, and G. Feng, "Reliable, $\mathrm{H}_{\infty}$ control for discrete-time piecewise linear systems with infinite distributed delays," Automatica, vol. 45, no. 12, pp. 2991-2994, 2009.

[51] Y. Liu, Z. Wang, J. Liang, and X. Liu, "Synchronization and state estimation for discrete-time complex networks with distributed delays," IEEE Transactions on Systems, Man, and Cybernetics Part B: Cybernetics, vol. 38, no. 5, pp. 1314-1325, 2008.

[52] V. B. Kolmanovskii and J.-P. Richard, "Stability of some linear systems with delays," IEEE Transactions on Automatic Control, vol. 44, no. 5, pp. 984-989, 1999.

[53] E. Fridman, "New Lyapunov-Krasovskii functionals for stability of linear retarded and neutral type systems," Systems and Control Letters, vol. 43, no. 4, pp. 309-319, 2001.

[54] S. Xu and J. Lam, "A survey of linear matrix inequality techniques in stability analysis of delay systems," International Journal of Systems Science, vol. 39, no. 12, pp. 1095-1113, 2008.

[55] M. S. Mahmoud and M. Zribi, " $\mathrm{H}_{\infty}$-controllers for time-delay systems using linear matrix inequalities," Journal of Optimization Theory and Applications, vol. 100, no. 1, pp. 89-122, 1999.

[56] B. D. O. Anderson and J. B. Moore, Optimal Filtering, Prentice Hall, New York, NY, USA, 1979.

[57] M. S. Mahmoud, N. F. Al-Muthairi, and S. Bingulac, "Robust Kalman filtering for continuous time-lag systems," Systems \& Control Letters, vol. 38, no. 4-5, pp. 309-319, 1999.

[58] M. S. Mahmoud, S. Z. Selim, and P. Shi, "Global exponential stability criteria for neural networks with probabilistic delays," IET Control Theory and Applications, vol. 4, no. 11, pp. 24052415, 2010.

[59] G. C. Walsh, H. Ye, and L. G. Bushnell, "Stability analysis of networked control systems," IEEE Transactions on Control Systems Technology, vol. 10, no. 3, pp. 438-446, 2002.

[60] S. Yin, L. Yu, and W.-A. Zhang, "A switched system approach to networked $H_{\infty}$ filtering with packet losses," Circuits, Systems, and Signal Processing, vol. 30, no. 6, pp. 1341-1354, 2011. 
[61] R. A. Gupta and M.-Y. Chow, "Networked control system: overview and research trends," IEEE Transactions on Industrial Electronics, vol. 57, no. 7, pp. 2527-2535, 2010.

[62] Y. Tipsuwan and M. Y. Chow, "Control methodologies in networked control systems," Control Engineering Practice, vol. 11, no. 10, pp. 1099-1111, 2003.

[63] W. Zhang, M. S. Branicky, and S. M. Phillips, "Stability of networked control systems," IEEE Control Systems, vol. 21, no. 1, pp. 84-99, 2001.

[64] S. Zampieri, "Trends in networked control systems," in Proceedings of the 17th World Congress, pp. 2886-2894, Seoul, Republic of Korea, July 2008.

[65] L. A. Montestruque and P. J. Antsaklis, "On the model-based control of networked systems," Automatica, vol. 39, no. 10, pp. 1837-1843, 2003.

[66] P. Seiler and R. Sengupta, "An $\mathrm{H}_{\infty}$ approach to networked control," IEEE Transactions on Automatic Control, vol. 50, no. 3, pp. 356-364, 2005.

[67] M. S. Mahmoud, H. N. Nounou, and Y. Xia, "Robust dissipative control for internet-based switching systems," Journal of the Franklin Institute. Engineering and Applied Mathematics, vol. 347, no. 1, pp. 154-172, 2010.

[68] P. Seiler and R. Sengupta, "Analysis of communication losses in vehicle control problems," in Proceedings of the American Control Conference, vol. 2, pp. 1491-1496, June 2001.

[69] F.-L. Lian, J. Moyne, and D. Tilbury, "Network design consideration for distributed control systems," IEEE Transactions on Control Systems Technology, vol. 10, no. 2, pp. 297-307, 2002.

[70] W. Zhang, M. S. Branicky, and S. M. Phillips, "Stability of networked control systems," IEEE Control Systems Magazine, vol. 21, no. 1, pp. 84-99, 2001.

[71] W.-A. Zhang and L. Yu, "Modelling and control of networked control systems with both network-induced delay and packetdropout," Automatica, vol. 44, no. 12, pp. 3206-3210, 2008.

[72] Y. Mikheev, V. Sobolev, and E. Fridman, "Asymptotic analysis of digital control systems," Automation and Remote Control, vol. 49, pp. 1175-1180, 1988.

[73] E. Fridman, A. Seuret, and J.-P. Richard, "Robust sampleddata stabilization of linear systems: an input delay approach," Automatica, vol. 40, no. 8, pp. 1441-1446, 2004.

[74] K. Liu and E. Fridman, "Wirtinger's inequality and Lyapunovbased sampled-data stabilization," Automatica, vol. 48, no. 1, pp. 102-108, 2012.

[75] P. Naghshtabrizi, J. P. Hespanha, and A. R. Teel, "Exponential stability of impulsive systems with application to uncertain sampled-data systems," Systems and Control Letters, vol. 57, no. 5, pp. 378-385, 2008.

[76] K. Liu, V. Suplin, and E. Fridman, "Stability of linear systems with general sawtooth delay," IMA Journal of Mathematical Control and Information, vol. 27, no. 4, pp. 419-436, 2010.

[77] K. Liu and E. Fridman, "Networked-based stabilization via discontinuous Lyapunov functionals," International Journal of Robust and Nonlinear Control, vol. 22, no. 4, pp. 420-436, 2012.

[78] M. S. Mahmoud, "Decentralized stabilization of interconnected systems with time-varying delays," IEEE Transactions on Automatic Control, vol. 54, no. 11, pp. 2663-2668, 2009.

[79] A. Seuret and F. Gouaisbaut, "Wirtinger-based integral inequality: application to time-delay systems," Automatica, vol. 49, no. 9, pp. 2860-2866, 2013.
[80] O. M. Kwon, M. J. Park, J. H. Park, S. M. Lee, and E. J. Cha, "Improved results on stability of linear systems with timevarying delays via Wirtinger-based integral inequality," Journal of the Franklin Institute. Engineering and Applied Mathematics, vol. 351, no. 12, pp. 5386-5398, 2014.

[81] M. J. Park, O. M. Kwon, J. H. Park, S. M. Lee, and E. J. Cha, "Stability of time-delay systems via Wirtinger-based double integral inequality," Automatica, vol. 55, pp. 204-208, 2015.

[82] O. M. Kwon, M. J. Park, J. H. Park, and S. M. Lee, "Improvement on the feasible region of $\mathrm{H}_{\infty}$ performance and stability for systems with interval time-varying delays via augmented Lyapunov-Krasovskii functional," Journal of the Franklin Institute. Engineering and Applied Mathematics, vol. 353, no. 18, pp. 4979-5000, 2016.

[83] O. M. Kwon, M. J. Park, J. H. Park, and S. M. Lee, "Stability and stabilization of T-S fuzzy systems with time-varying delays via augmented Lyapunov-Krasovskii functionals," Information Sciences, vol. 372, pp. 1-15, 2016.

[84] H. Dong, Z. Wang, and H. Gao, "Robust $H_{\infty}$ filtering for a class of nonlinear networked systems with multiple stochastic communication delays and packet dropouts," IEEE Transactions on Signal Processing, vol. 58, no. 4, pp. 1957-1966, 2010.

[85] Y. Liu, Z. Wang, and W. Wang, "Robust reliable control for discrete-time-delay systems with stochastic nonlinearities and multiplicative noises," Optimal Control Applications and Methods, vol. 32, no. 3, pp. 285-297, 2011. 


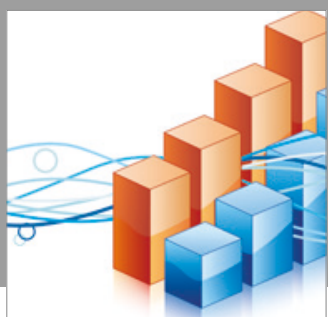

Advances in

Operations Research

vatersals

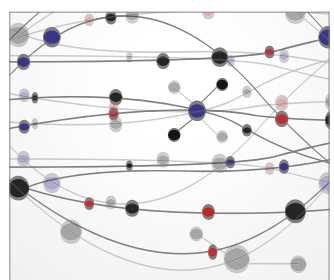

\section{The Scientific} World Journal
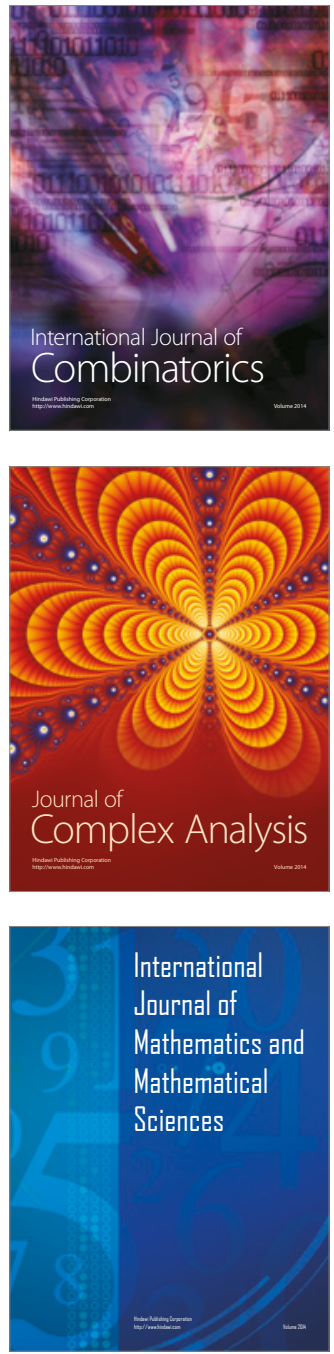
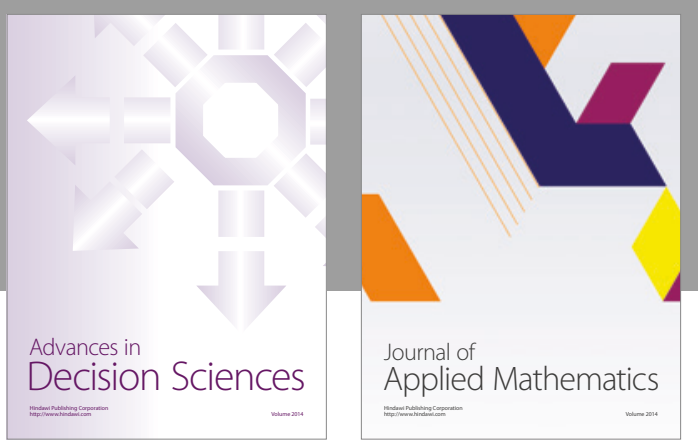

Algebra

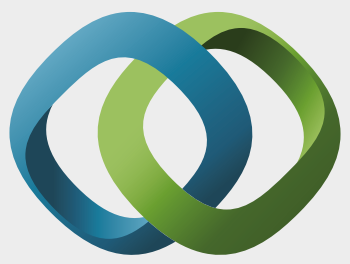

\section{Hindawi}

Submit your manuscripts at

https://www.hindawi.com
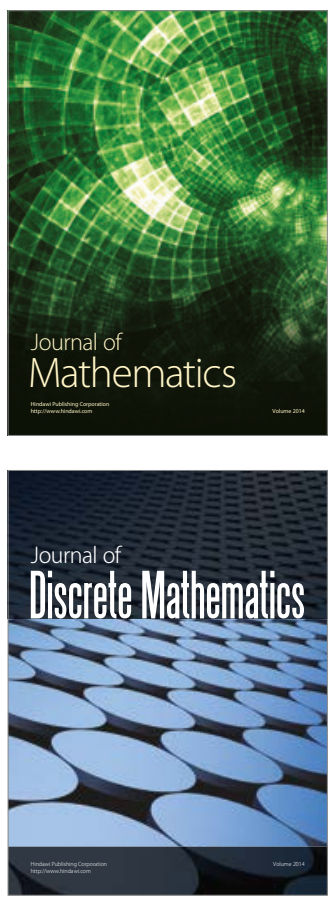

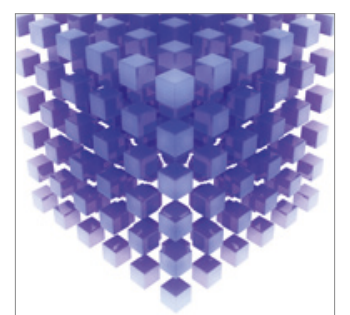

Mathematical Problems in Engineering
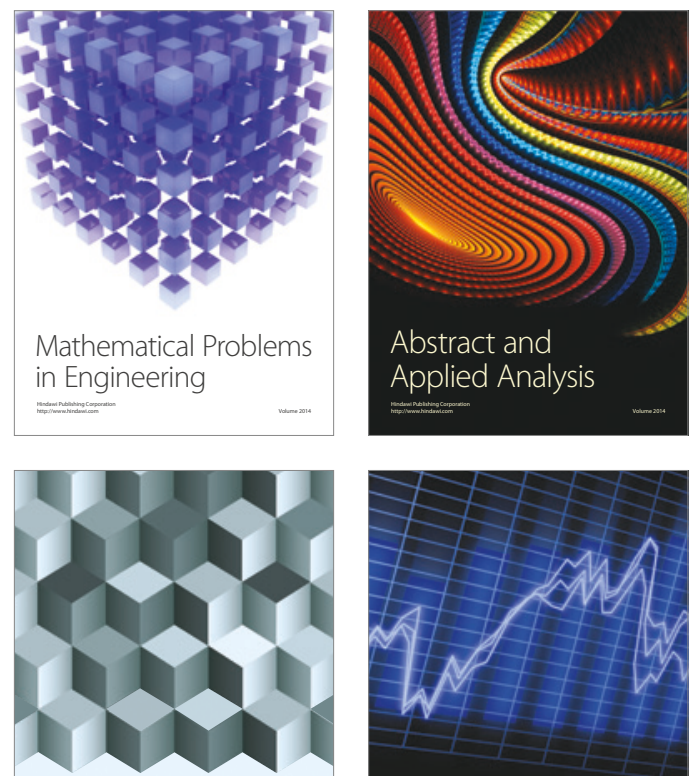

Journal of

Function Spaces

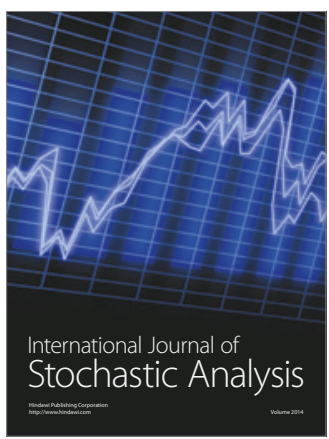

Probability and Statistics
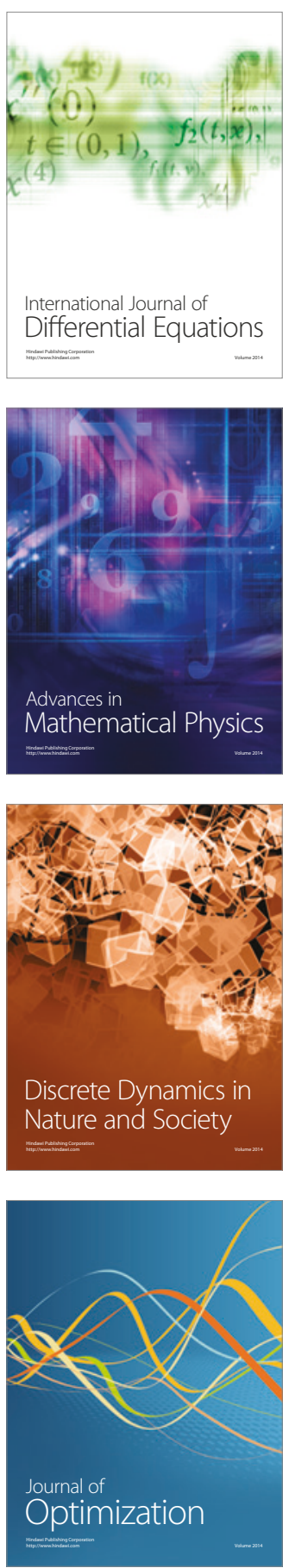